\title{
Performance of Deconvolution Methods in Estimating CBOC-Modulated Signals
}

\author{
Danai Skournetou, ${ }^{1}$ Ali H. Sayed, ${ }^{2}$ and Elena Simona Lohan ${ }^{1}$ \\ ${ }^{1}$ Departartment of Communications Engineering, Tampere University of Technology, Tampere 33720, Finland \\ ${ }^{2}$ Department of Electrical Engineering, University of California, Los Angeles, CA 90095, USA
}

Correspondence should be addressed to Danai Skournetou, danai.skournetou@tut.fi

Received 3 November 2010; Revised 8 March 2011; Accepted 11 April 2011

Academic Editor: Olivier Julien

Copyright (C) 2011 Danai Skournetou et al. This is an open access article distributed under the Creative Commons Attribution License, which permits unrestricted use, distribution, and reproduction in any medium, provided the original work is properly cited.

\begin{abstract}
Multipath propagation is one of the most difficult error sources to compensate in global navigation satellite systems due to its environment-specific nature. In order to gain a better understanding of its impact on the received signal, the establishment of a theoretical performance limit can be of great assistance. In this paper, we derive the Cramer Rao lower bounds (CRLBs), where in one case, the unknown parameter vector corresponds to any of the three multipath signal parameters of carrier phase, code delay, and amplitude, and in the second case, all possible combinations of joint parameter estimation are considered. Furthermore, we study how various channel parameters affect the computed CRLBs, and we use these bounds to compare the performance of three deconvolution methods: least squares, minimum mean square error, and projection onto convex space. In all our simulations, we employ CBOC modulation, which is the one selected for future Galileo E1 signals.
\end{abstract}

\section{Introduction}

In order for a user to compute their three-dimensional position and to correct the clock offset, the distance between its GNSS receiver and at least four satellites is required. Mass market receivers of code division multiple access(CDMA-) based positioning compute the unknown distance (also known as pseudorange) by estimating the total code delay.

Apart from the propagation delay, the signal undergoes a variety of channel distortions (such as those caused by ionosphere and troposphere layers) which introduce further delays [1]. Multipath propagation is a major source of error in the range measurement, because it can significantly delay the signal and it cannot be mitigated with differential methods due to its site-specific nature [2]. Environments prone to multipath effects are densely built areas or areas with large obstacles, which are typically encountered in metropolitan areas, where the concentration of GNSS users is high. If the receiver does not estimate the multipath delay with sufficient accuracy, then it suffers a degradation in the accuracy of range estimation and an increase in the processing time [3].
The distortion effects of multipath propagation have been known to the GNSS community for long time, and several efforts to mitigate them have taken place. A large portion of these efforts has been focused on the tracking stage of a receiver where fine estimates of the line-ofsight (LOS) code delay and carrier phase are required. One of the most commonly used code tracking structures are the so-called Delay locked loops (DLLs), which belong to the category of feedback estimators. Examples of such structures include the popular narrow correlator [4], double delta correlator [5], strobe and edge correlators [6], highresolution correlator and other optimized multiple gate delay (MGD) structures [7]. However, the feedback-based estimators are generally sensitive to closely spaced path scenarios and potential acquisition errors. As an alternative solution, various feedforward approaches have been proposed in the literature [8]. While improving the delay estimation accuracy, these approaches typically require more correlators than DLL-based ones and are sensitive to the noise-dependent threshold choice. Various combinations of feedback and feedforward approaches aim at improved accuracy $[9,10]$. 


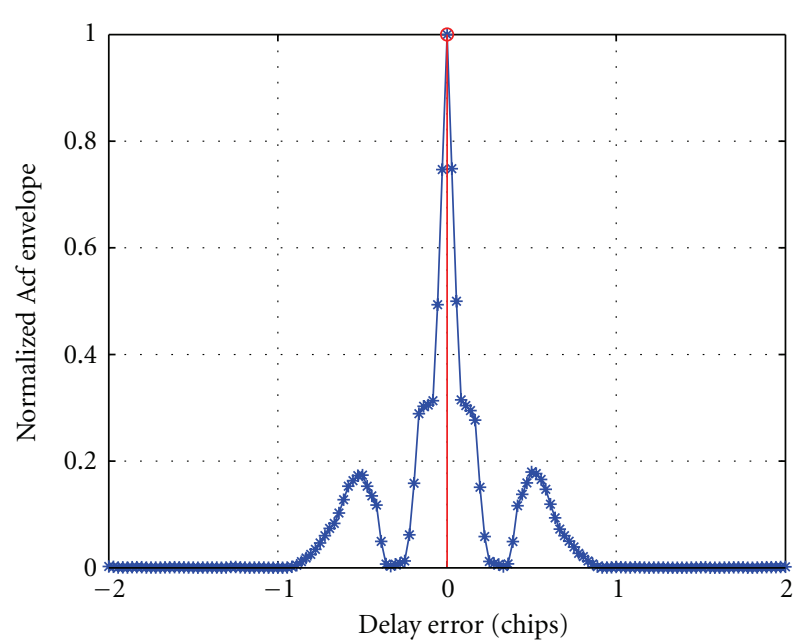

(a)

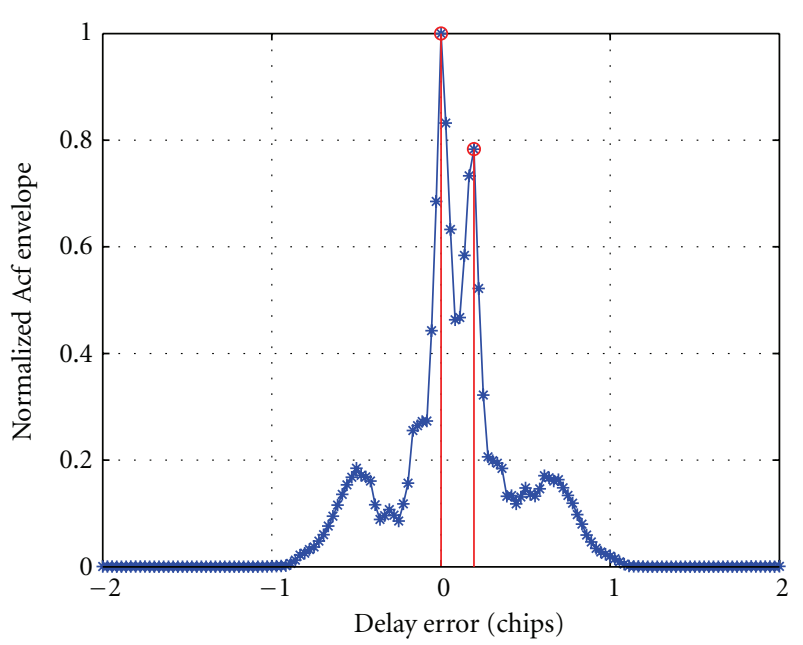

(b)

FIgUre 1: Normalized Acf envelope of CBOC(-) modulated signal for single- (a) and two-path (b) channel (no noise, infinite bandwidth).

In the carrier tracking stage, multipath mitigation has been a challenging problem as well. Carrier phase multipath has been commonly studied in 2-path channels using a phasor diagram that illustrates the relation between the phase of the LOS signal and the multipath [11-13]. In [14], a geometric perspective is employed that involves different configurations of the antenna-reflector(s) geometry. Other methods include the ashtech enhanced Strobe correlator [15] and the multipath estimating delay lock loop (MEDLL); the latter jointly estimates the delay, relative amplitude, and phase parameters of the direct and multipath signals based on the maximum likelihood theory [16]. Both are advanced techniques with improved performance in long delay multipath errors; however, they are heavily covered by patents.

1.1. BOC Modulation. Towards the end of the 1990s, a new modulation technique, called binary offset carrier (BOC), was recommended for future GNSS signals for achieving sufficient spectral separation with existing GPS signals [17]. Moreover, because the width of the main lobe in the envelope of the autocorrelation function (Acf) is narrower than the one in binary phase shift Key (BPSK) modulated signals (i.e., used in GPS C/A signal), improved tracking accuracy could be achieved. There have been several variants of BOC suggested in the literature for different signal types included in the GPS modernisation plans and Galileo specifications. Among those variants, Sine-BOC $(1,1)$ was initially used in the standards for the L1 open service (OS) Galileo signals, but afterwards multiplexed BOC (MBOC) was selected [18]. MBOC is a weighted combination of sine- $\operatorname{BOC}(1,1)$ and sine-BOC $(6,1)$ components (we notice that in the notation $\operatorname{BOC}(m, n), m$ is the ratio of the sub-carrier frequency over the reference frequency, of $1.023 \mathrm{MHz}, n$ is the ratio of the chip rate over the reference frequency and the ratio $2 \mathrm{~m} / \mathrm{n}$ describes the BOC order) and is defined as a common spectrum to be matched by both the Galileo and the GPS L1/E1 OS signals. The MBOC spectrum can be realized in the time domain with many different approaches and the two chosen for GPS and Galileo are (1) time multiplexed BOC (TMBOC) and (2) composite BOC (CBOC), respectively.

In the first implementation, the whole signal is divided into blocks of $N$ code symbols, and $M<N$ of $N$ code symbols are sine-BOC $(1,1)$ modulated, while $N-M$ code symbols are sine-BOC $(6,1)$ modulated. In the CBOC implementation, we have a weighted combination of Sine$\operatorname{BOC}(1,1)$ and Sine-BOC $(6,1)$ modulated code symbols. When the combination is an addition of the two components, we have the so-called CBOC ("+"), and when we subtract the sine-BOC $(6,1)$ part from the sine- $\operatorname{BOC}(1,1)$ part, we have the so-called CBOC ("-") type of modulation. The CBOC ("+") scheme is used in the implementation of the Galileo OS data channel, while CBOC ("-") is used in the pilot channel [18]. The normalized envelope of autocorrelation function of a CBOC (“-”) modulated signal can be seen in the left plot of Figure 1.

While BOC modulation improves the tracking accuracy, it introduces an extra challenge in the tracking stage. More precisely, the additional peaks in the Acf, the number of which depends on the BOC type, increase the probability of tracking the wrong peak. In the right plot of Figure 1, we can see how the Acf is distorted due to the presence of a second path (located on the right side of the first path, 7 samples far from it). If the tracking module is falsely locked in the second peak, then code delay error is produced. One can now envision how more complex the Acf would look like in the presence of more paths and in the case of fading channel.

1.2. Motivation and Contribution. While there is an ample number of scientific works related to tracking of BPSKmodulated signals, the amount of studies focusing on CBOC modulated signals is significantly smaller, mostly because it was relatively recently selected for the Galileo OS. Examples of existing work include $[19,20]$ which compare the tracking performance of various discriminators for the 


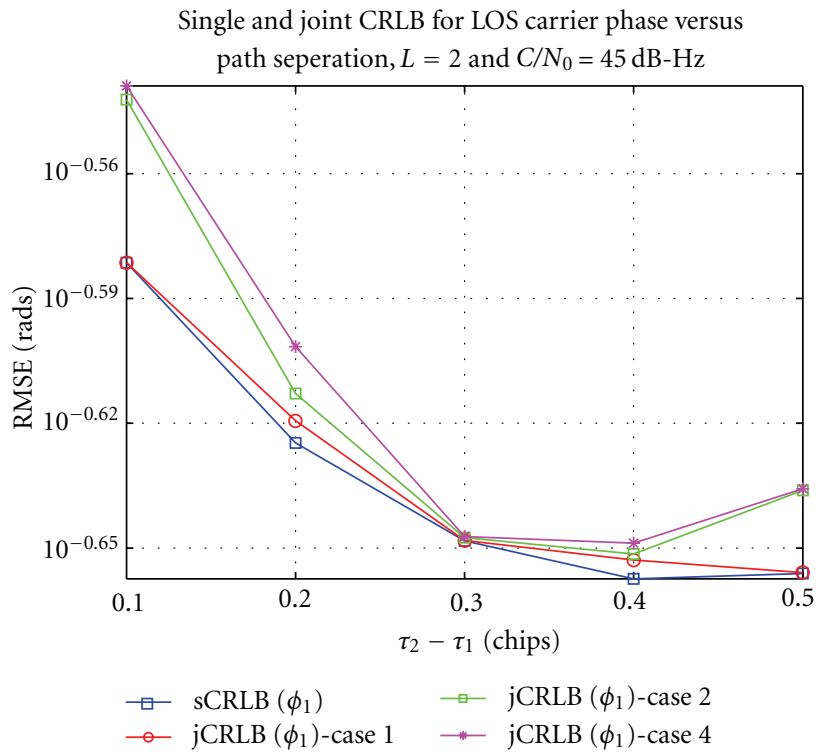

(a)

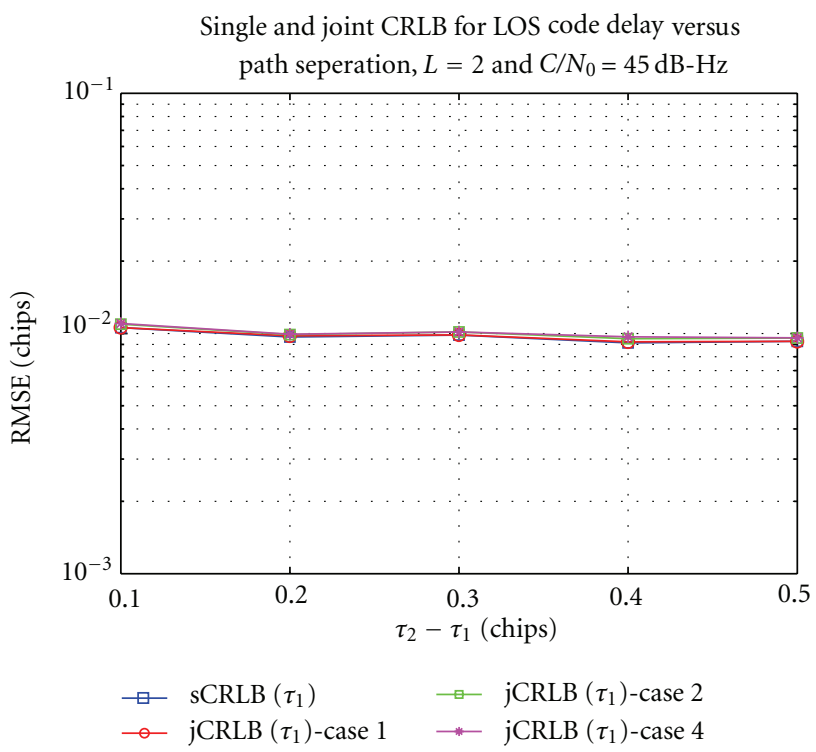

(b)

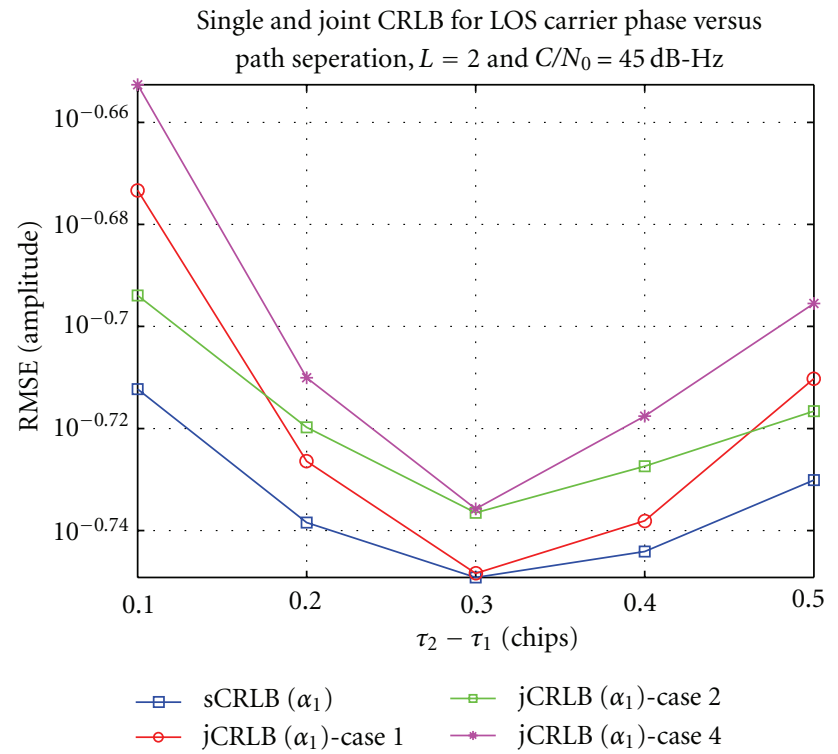

(c)

FIGURE 2: RMSE of single and joint CRLB versus path separation $\left(\tau_{2}-\tau_{1}\right)$ for LOS carrier phase (a), code (b) delay and amplitude (c), $L=2$ and $\mathrm{C} / \mathrm{N}_{0}=45 \mathrm{~dB}-\mathrm{Hz}$.

new modulation schemes, such as CBOC and TMBOC. In [21], the authors study the impact of the new modulation schemes on carrier tracking loop and in [22] the effects of heavy multipath propagation in combination with $\mathrm{CBOC}$ modulation are examined.

A thorough literature review reveals that the majority of existing work that adopts CBOC modulation studies the performance of mainly state-of-the-art code discriminators, such as early-minus-late, dot product, and Strobe correlator, while carrier phase estimation has been much less studied. In this paper, we are interested in the performance of less popular algorithms but which are used to estimate all three parameters of CBOC-modulated signals (i.e., carrier phase, code delay, and amplitude). In particular, we study the performance of deconvolution methods which are means of inverse filtering. One of the adverse effects of inverse filtering, when noise is present, is the noise enhancement. The noise enhancement effect can be reduced by using the socalled constrained inverse filtering methods. These methods are constrained in the sense that they do not allow the output values to lie outside some predefined set or in the sense that the inverse operator is never completely formed but only approximated iteratively. Among the constrained inverse filtering methods, the best known ones are the leastsquares (LS) techniques and the projection onto convex sets (POCS) algorithm $[23,24]$. 
A commonly used method for assessing the performance of an estimator is to compare its error variance with the theoretically minimum attainable, the latter of which is known as the Cramer Rao lower bound (CRLB) [25]. While the methodology for deriving the CRLB is straightforward and has been reported in [25], it always has to be tailored to the estimation problem in question (i.e., different estimation problems are encountered in different research areas; therefore depending, on the environment, each estimation problem is positioned, the assumptions made, and the parameters of interest may differ).

Based on the above discussion, the objectives of this paper are formed as follows: first, to provide a theoretical model that leads to the CRLB for the signal's unknown parameter vectors of carrier phase, code delay, and amplitude by taking into account the multipath effects and the correlated noise at the output of the correlators and receiver filters. More precisely, we present two types of bounds. The first one, called single CRLB (sCRLB), represents the CRLB for a single parameter vector (i.e., a vector containing the unknown parameter for each path), where we assumed that the remaining parameters are known or perfectly estimated. The second type, called joint CRLB (jCRLB), reveals the theoretical limits given that a set of parameter vectors is jointly estimated. The reason for distinguishing between single and joint CRLB is that by comparing them, we can gain meaningful information related to the importance of each parameter in the estimation accuracy of the other set of parameters. The computations assume a static multipath channel with arbitrary number of paths and additive white Gaussian noise (AWGN). We use static channels, because we want to examine the minimum achievable performance and because modeling the phases in fading channels introduces additional errors. However, the model can be easily adapted for fading channels by taking into account the statistical characteristics of the profile at hand.

The second objective is to analyze theoretically the impact of different channel parameters such as $C / N_{0}$, path separation, and number of channel paths on the estimator accuracy bounds. Finally, we provide performance comparisons between the derived theoretical limits and a set of deconvolution estimators, namely, least squares (LS), minimum mean square error (MMSE) and a POCS algorithm proposed earlier by the authors [24].

The remainder of this paper is organized as follows: Section 2 presents the system model. Section 4 describes the simulation setup and includes the results and the discussion. Finally, Section 5 summarizes the findings of this paper (the detailed derivation of the CRLBs can be found in the appendix).

\section{System Model}

The satellite transmitted signal, $x(t)$, can be modeled as the convolution between the modulating waveform $s_{\mathrm{CBOC}}(t)$, the pseudorandom (PRN) CDMA code, and the modulated data as [26]

$$
x(t)=s_{\mathrm{CBOC}}(t) \star \sum_{n=-\infty}^{+\infty} \sum_{g=1}^{S_{F}} b_{n} c_{g, n} \delta\left(t-n T-g T_{c}\right)
$$

where $\star$ is the convolution operator, $b_{n}$ is the $n$th complex data symbol (in case of a pilot channel, it is equal to 1 ), $T$ is the symbol period, $c_{g, n}$ is the $g$ th chip corresponding to the $n$th symbol, $T_{c}$ is the chip period, $S_{F}$ is the spreading factor $\left(S_{F}=T_{\text {sym }} / T_{c}\right), \delta(t)$ is the Dirac pulse, and $s_{\text {CBOC }}(t)$ stands for the CBOC modulated signal.

After the signal is transferred to the passband, it is transmitted over a multipath static or multipath fading channel, where all interference sources (except for the multipaths) are lumped into a single additive Gaussian noise term. At the receiver side, the signal is downconverted to the baseband, and it can be written as

$$
r_{x}(t)=\sqrt{E_{b}} \sum_{l=1}^{L} \alpha_{l} x\left(t-\tau_{l}\right) e^{j\left(2 \pi f_{D} t+\phi_{l}\right)}+\eta(t),
$$

where $E_{b}$ is the data bit energy, $\alpha_{l}, \tau_{l}$, and $\phi_{l}$ are the amplitude, code delay, and carrier phase offset of the $l$ th path, respectively, $L$ is the number of channel paths, $f_{D}$ is the Doppler shift introduced by the channel, and $\eta(t)$ is the additive Gaussian noise of zero mean and double-sided power spectral density $N_{0} \mathrm{~W} / \mathrm{Hz}$.

After downconverting the received signal and correlating it with the reference modulated PRN code (stored in the receiver), we get [24]

$$
y_{R}(t)=\sqrt{E_{b}} \sum_{l=1}^{L} \alpha_{l} R_{m}\left(\tau_{i}, \tau_{q}\right) e^{-j\left(2 \pi \Delta_{f_{D}} t+\phi_{l}\right)}+v(t),
$$

where $\Delta_{f_{D}}$ is the Doppler shift error (i.e., a residue of the acquisition stage), $v(t)$ is the complex colored Gaussian noise of the despread signal with zero mean and covariance matrix $\mathbf{C}_{v}=\sigma^{2} \mathbf{H}$, where $\sigma^{2}$ is the variance per each correlator, equal to the two-sided power spectral density of $N_{0} / 2 \mathrm{~W} / \mathrm{Hz}$, and $\mathbf{H}$ is the correlation matrix given in (6) (i.e., independent of the unknown parameters) [27]. Moreover, $R_{m}\left(\tau_{i}, \tau_{q}\right)$ is the ideal continuous autocorrelation function of the modulated code at delays $\tau_{i}$ and $\tau_{q}$, which is expressed as

$$
R_{m}\left(\tau_{i}, \tau_{q}\right)=\mathbf{E}\left(\frac{1}{T_{\mathrm{symb}}} \int_{(m-1) T_{\mathrm{symb}}}^{m T_{\mathrm{symb}}} x\left(t-\tau_{i}\right) x\left(t-\tau_{q}\right)\right)
$$

where $\mathbf{E}(\cdot)$ is the expectation operation, $m$ is the code epoch index, and $T_{\text {symb }}$ is the symbol duration.

Assuming that the Doppler shift has been successfully removed (i.e., $\Delta_{f_{D}}=0$ ), we can transform (3) into [24]

$$
\mathbf{y}=\mathbf{H w}(\tau, \boldsymbol{\alpha}, \phi)+\mathbf{v}
$$




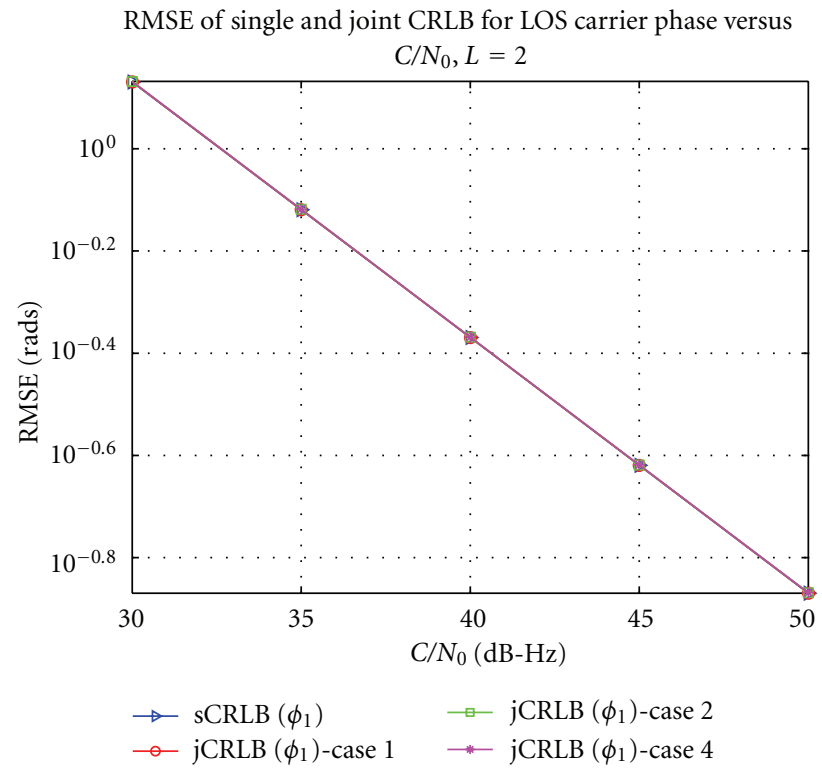

(a)

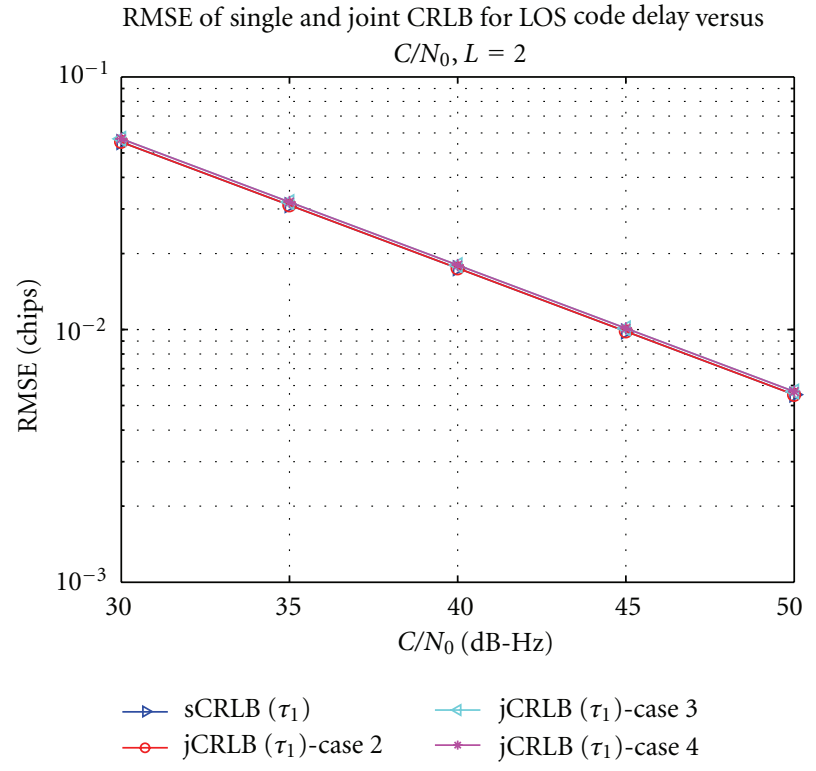

(b)

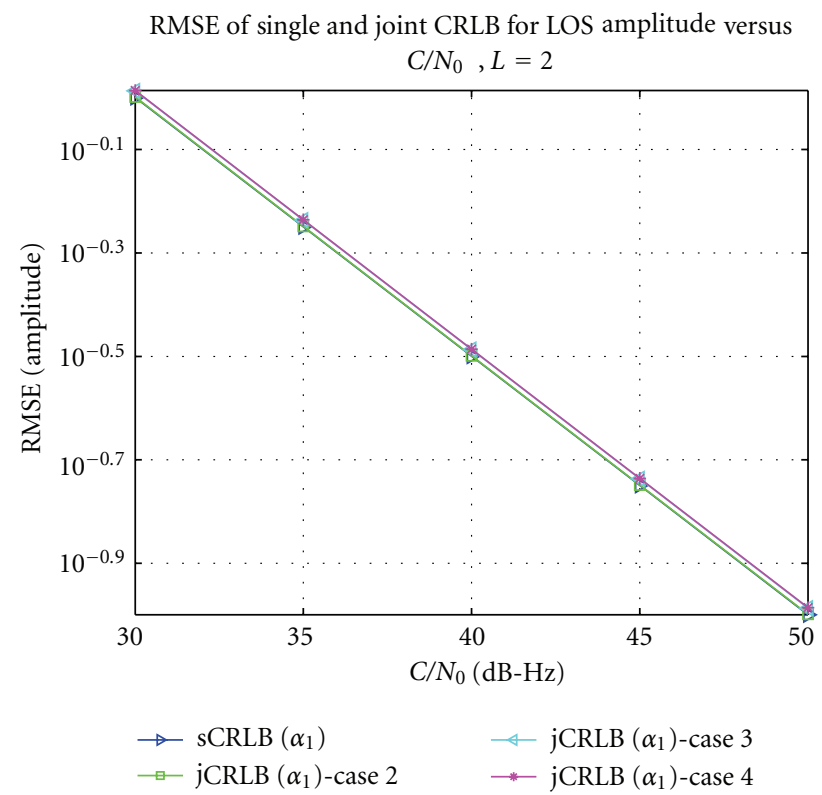

(c)

Figure 3: RMSE of Single and Joint CRLB versus $C / N_{0}$ for LOS carrier phase (a), code (b) delay and amplitude (c).

where $\mathbf{y}$ is the data column vector that contains the complex correlation output sampled at rate $N_{s}$ and $\mathbf{H}$ is the pulse shape deconvolution matrix of size $K \times K$ given by

$$
\mathbf{H}=\left[\begin{array}{ccc}
h\left(\tau_{1}, \tau_{1}\right) & \cdots & h\left(\tau_{1}, \tau_{K}\right) \\
\vdots & \ddots & \vdots \\
h\left(\tau_{K}, \tau_{1}\right) & \cdots & h\left(\tau_{K}, \tau_{K}\right)
\end{array}\right],
$$

where $h\left(\tau_{i}, \tau_{q}\right)$ is the output of the ideal discrete autocorrelation function at code delays $\tau_{i}$ and $\tau_{q}$ for $1 \leq i \leq K$ and $1 \leq q \leq K$, respectively. The mathematical expression can be found from (4), by substituting the integral with a summation and the continuous time $t$ with the discrete sampled time instances). In addition, the term $\tau_{K}$ is the maximum delay spread of the channel (i.e., $\tau_{K}=\tau_{\max } T_{s}$, where $T_{s}$ is the sampling period and $\tau_{\max }$ is the maximum delay). The noise vector $\mathbf{v}$ contains the complex colored Gaussian noise terms of the despread signal with zero mean and covariance matrix $\mathbf{C}_{v}$. The unknown vector, $\mathbf{w}$, is a function of the signal parameter vectors $\boldsymbol{\tau}, \boldsymbol{\alpha}$, and $\phi$ that we want to estimate. Specifically, the elements of the unknown vector $\mathbf{w}$ have the following interpretation: ideally (i.e., in noise-free conditions), if a path is present at delay $\tau_{k}$, then $w_{k}$ would be equal to $a_{k} e^{j \phi_{k}}$; otherwise, $w_{k}$ is zero. In 
other words, the positions of the nonzero elements in $\mathbf{w}$ correspond to the delay of the channel paths and the value of the nonzero element contains the amplitude and phase information. Thus, in order to find the unknown signal parameters, we first need to locate the nonzero elements of the $\mathbf{w}$.

The above formulation of the model transforms the problem into solving a system of linear equations. Several methods have been proposed for solving such a system (see Section 1). In what follows, this model constitutes the basis for deriving the theoretically achievable limits of the parameters of interest.

\section{Theoretical Estimation Limits}

A commonly used method for assessing the performance of an unbiased estimator is to compare its error variance with the Cramer Rao lower bound (CRLB) [25]. The computation of CRLB requires that the probability density function ( $\mathrm{pdf}$ ) of the observed data is known. However, because in our case the observed data are contaminated by colored Gaussian noise, we perform a whitening process so as to transform the noise into additive white Gaussian noise (AWGN) whose pdf is known.

In [27], the covariance matrix $\mathbf{C}_{v}$ is found to be equal to $\sigma^{2} \mathbf{H}$, which is independent of the unknown parameters. Furthermore, it can be shown that $\mathbf{C}_{v}$ is positive definite; therefore, it can be factored as $\mathbf{C}_{v}^{-1}=\left(1 / \sigma^{2}\right) \mathbf{H}^{-1}=\mathbf{D D}^{*}$, where $\mathbf{D}$ is a lower triangular, $K \times K$ invertible matrix, and $D^{*}$ denotes the conjugate of $\mathbf{D}$. According to [25], the matrix $\mathbf{D}$ can act as a whitening transformation when applied to $\mathbf{v}$. Multiplying the terms of (5) leftwise with $\mathbf{D}$ gives

$$
\begin{gathered}
\operatorname{Dy}=\operatorname{DHw}(\tau, \alpha, \phi)+\operatorname{Dv} \\
\tilde{\mathbf{y}}=\tilde{\mathbf{H}} \mathbf{w}(\tau, \alpha, \phi)+\tilde{\mathbf{v}}=\mathbf{s}(\tau, \alpha, \phi)+\tilde{\mathbf{v}}
\end{gathered}
$$

where $\tilde{\mathbf{v}}$ is now AWGN with zero mean and unit variance [25] and $\boldsymbol{s}(\boldsymbol{\tau}, \boldsymbol{\alpha}, \phi)$ is

$$
\mathbf{s}(\boldsymbol{\tau}, \boldsymbol{\alpha}, \phi)=\left[\sum_{l=1}^{L} \tilde{h}_{\tau_{1}, \tau_{l}} \alpha_{l} e^{j \phi_{l}} \ldots \sum_{l=1}^{L} \tilde{h}_{\tau_{K}, \tau_{l}} \alpha_{l} e^{j \phi_{l}}\right]^{T},
$$

where $\tilde{h}$ is used to describe the elements of the matrix $\widetilde{\mathbf{H}}$. Assuming that the unknown vector parameter to be estimated is denoted by $\boldsymbol{\theta}$, we can write the pdf as [25]

$$
p(\tilde{\mathbf{y}} ; \boldsymbol{\theta})=\frac{1}{\left(2 \pi \sigma^{2}\right)^{k / 2}} \exp \left\{-\frac{1}{2 \sigma^{2}} \sum_{k=1}^{K}|\tilde{y}(k)-s(\boldsymbol{\theta} ; k)|^{2}\right\},
$$

where in the case of single CRLB, $\boldsymbol{\theta}$ is equal to one of the three parameter vectors, $\boldsymbol{\tau}, \boldsymbol{\alpha}$, or $\phi$, and in the case of joint CRLB, $\boldsymbol{\theta}$ is equal to any of the four possible combinations of the three parameter vectors (i.e., $\boldsymbol{\theta}=[\phi ; \boldsymbol{\tau}], \boldsymbol{\theta}=[\phi ; \boldsymbol{\alpha}], \boldsymbol{\theta}=[\boldsymbol{\tau} ; \boldsymbol{\alpha}]$ or $\boldsymbol{\theta}=[\phi ; \boldsymbol{\tau} ; \boldsymbol{\alpha}])$. Because (9) shows the dependency of the pdf upon the unknown parameter; it is termed the likelihood function [25].

\begin{tabular}{|c|c|c|c|}
\hline & Phase & Delay & Amplitude \\
\hline Single CRLB & $\operatorname{var}(\hat{\phi})$ & $\operatorname{var}(\hat{\boldsymbol{\tau}})$ & $\operatorname{var}(\widehat{\boldsymbol{\alpha}})$ \\
\hline Joint CRLB - Case 1 & $\operatorname{var}_{1, \text { joint }}(\hat{\phi})$ & $\operatorname{var}_{1, \text { joint }}(\hat{\boldsymbol{\tau}})$ & \\
\hline Joint CRLB - Case 2 & $\operatorname{var}_{2, \text { joint }}(\hat{\phi})$ & & $\operatorname{var}_{2, \text { joint }}(\widehat{\boldsymbol{\alpha}})$ \\
\hline Joint CRLB - Case 3 & & $\operatorname{var}_{3, \text { joint }}(\hat{\boldsymbol{\tau}})$ & $\operatorname{var}_{3, \text { joint }}(\widehat{\boldsymbol{\alpha}})$ \\
\hline Joint CRLB- Case 4 & $\operatorname{var}_{4, \text { joint }}(\hat{\phi})$ & $\operatorname{var}_{4, \text { joint }}(\hat{\boldsymbol{\tau}})$ & $\operatorname{var}_{4, \text { joint }}(\widehat{\boldsymbol{\alpha}})$ \\
\hline
\end{tabular}

If we assume that the estimator $\hat{\boldsymbol{\theta}}$ is unbiased and that the pdf satisfies the regularity condition, the single and joint
TABLE 1: Single and Joint CRLBs notations.

CRLBs can be derived in a straightforward manner (see the appendix for the detailed computations and Table 1 for the CRLBs notations used in this paper).

\section{Simulation Profiles and Results}

In the first part of simulations, we study the CRLB behavior of LOS signal versus various channel parameters. The signal was modulated using CBOC ("-") modulation (i.e., the modulation selected in the standards for future Galileo OS pilot signals [18]) and for the channel modeling we employ a decaying power delay profile (PDP) [28], meaning that $\overline{\alpha_{l}}=\overline{\alpha_{1}} e^{-\zeta \mathrm{PDP}\left(\tau_{l}-\tau_{1}\right)}$, where $\overline{\alpha_{1}}$ is the average amplitude of the 1st path and $\zeta_{\mathrm{PDP}}$ is the power decaying profile coefficient (assumed in the simulations to be equal to 0.09 when the path delays are expressed in samples). The carrier phase of each path was assumed to be uniformly distributed between $-\pi$ and $\pi$. At the receiver side, the bandwidth was assumed to be infinite, the sampling rate $f_{s}$ is equal to $N_{s} f_{c}$, where $N_{s}$ is oversampling factor and equal to 4 and $f_{c}$ is the chip rate (equal to $1.023 \mathrm{MHz}$ for L1 OS signals). Also, the coherent integration time $\left(N_{c}\right)$ was $1 \mathrm{~ms}$ (the equivalent $C / N_{0}$ after coherent and noncoherent integration is $C / N_{0, \text { eq. }}=$ $C / N_{0}+10 \log _{10}\left(N_{c} * \operatorname{sqrt}\left(N_{\mathrm{nc}}\right)\right)$. For the computation of the CRLBs, we have assumed that the bit energy is 1 and the noise variance $\left(\sigma^{2}\right)$ is then equal to $1 /\left(N_{c} N_{\mathrm{nc}} E_{b} / N_{0}\right)$, where $E_{b} / N_{0}$ is the energy per bit to noise power spectral density ratio. Moreover, we consider only the case of static multipath channels, because we wanted to examine the maximum achievable performance and because modeling the phase changes in fading channels introduces additional errors. In cases where we deviate from these values or needed additional parameters, we note this in the title and/or caption of the figures. As the performance metric, we use the root mean square error (RMSE) which is computed of 5000 random channel realizations.

In Figure 2, we see how the CRLBs vary with increasing path separation (i.e., $\tau_{2}-\tau_{1}$ ) and in the case when there are two paths and $C / N_{0}=45 \mathrm{~dB}-\mathrm{Hz}$ (we notice that all the $\mathrm{C} / \mathrm{N}_{0}$ values mentioned are the ones prior to any integration). For the case of carrier phase parameter (top left plot), we see that when the path separation is 0.3 chips the RMSE for single and joint CRLBs coincide. With respect to the code delay parameter, we see that the difference among single and joint CRLBs is minor, while in the case of amplitude, the differences are evident.

Figure 3 shows how the single and joint CRLBs behave with increasing $C / N_{0}$ in case of two-path channel. In this 


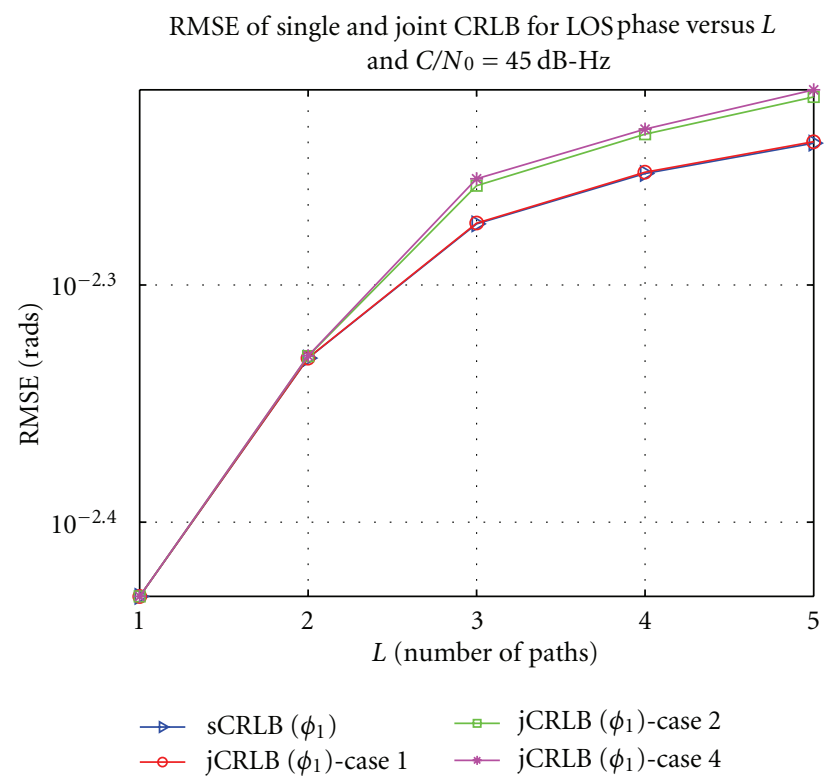

(a)
RMSE of single and joint CRLB for LOS code delay versus $L$ and $\mathrm{C} / \mathrm{N}_{0}=45 \mathrm{~dB}-\mathrm{Hz}$

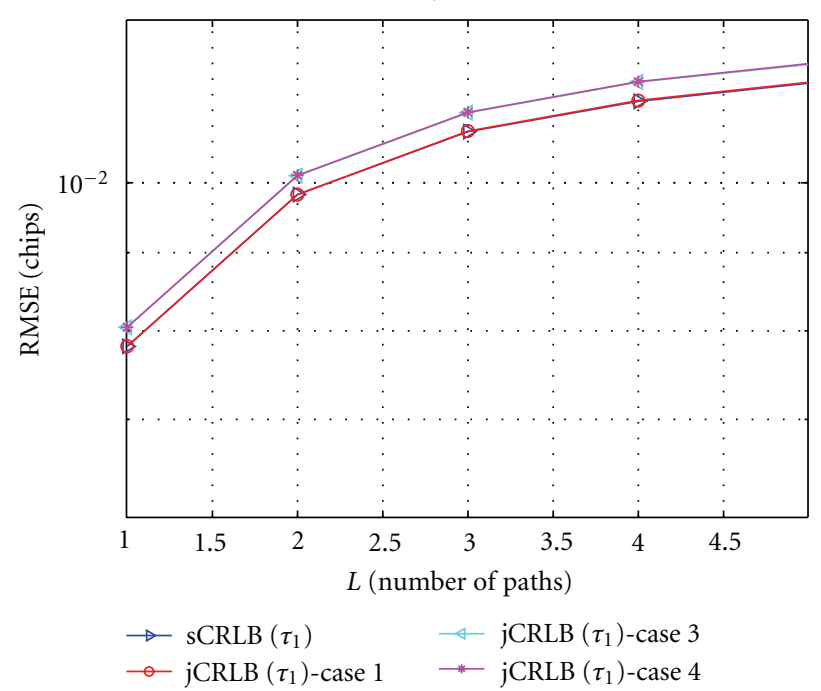

(b)

RMSE of single and joint CRLB for LOS amplitude versus $L$ and $\mathrm{C} / \mathrm{N}_{0}=45 \mathrm{~dB}-\mathrm{Hz}$

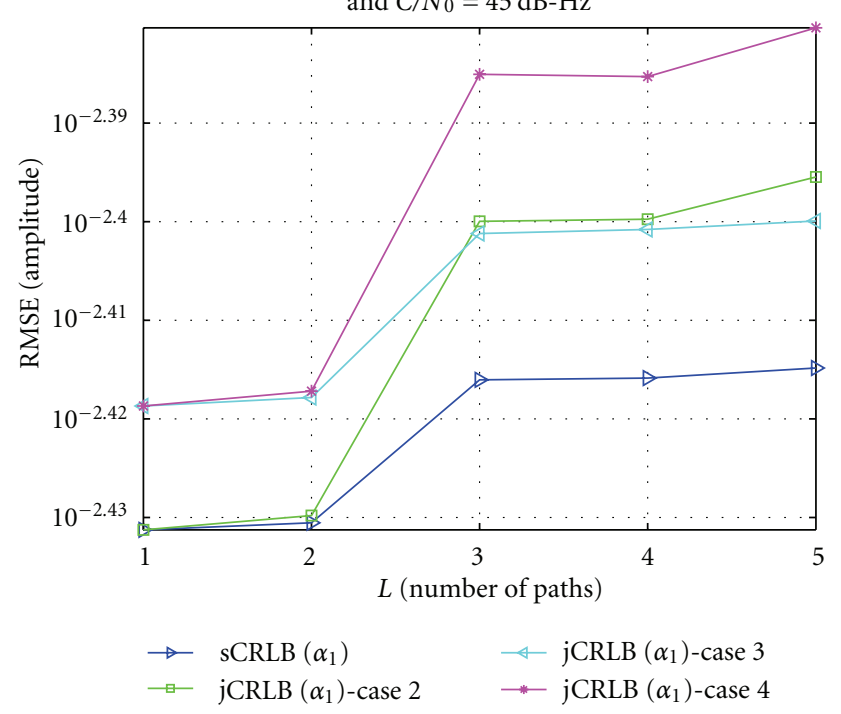

(c)

FIgURE 4: RMSE of Single and Joint CRLB versus number of paths, $C / N_{0}=45 \mathrm{~dB}-\mathrm{Hz}$.

case, the path separation was fixed to 0.3 chips, because according to Figure 2, this is the value when all CRLBs types behave the closest (thus, we can isolate the impact of $C / N_{0}$ ). From all three plots, we observe there are very slight differences between single and joint CRLBs. So, in two-path channels when, for example, we try to estimate all three synchronization parameters, we can achieve the same theoretical limit as when estimating only the LOS code delay and assuming the rest to be known.

Now, we are interested in studying the impact of the number of channel paths on the theoretically attainable bounds. Therefore, we used the same channel model with the previous scenario, only that now the $C / N_{0}$ was fixed to $45 \mathrm{~dB}$ $\mathrm{Hz}$ and the path separation 0.3 chips. From the top left plot of Figure 4 we notice that when the number of paths is one or two, there is no difference among single and joint CRLBs. When the number of paths increases, estimating all three parameters leads to the same limit as in the case of estimating jointly the phase and the amplitude. Common behavior for $L>2$ is also noticed for the case of single CRLB and the first case of joint. Regarding the code delay parameter, we notice that the similar performance between sCRLB and jCRLBCase 1 and between jCRLB-Case 3 and jCRLB_Case 4 is evident for all number of channel paths. 


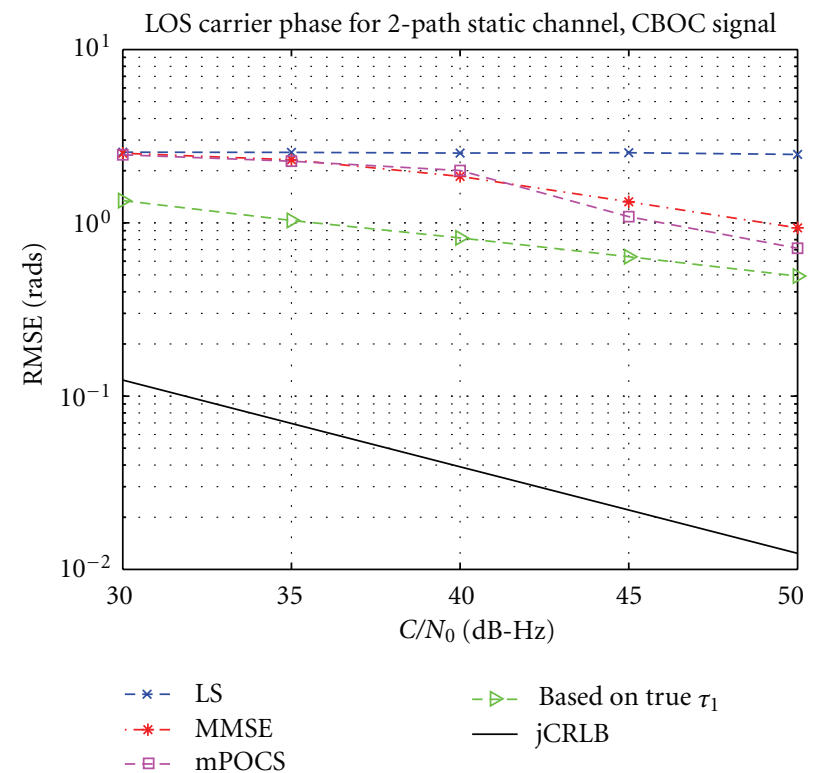

(a)

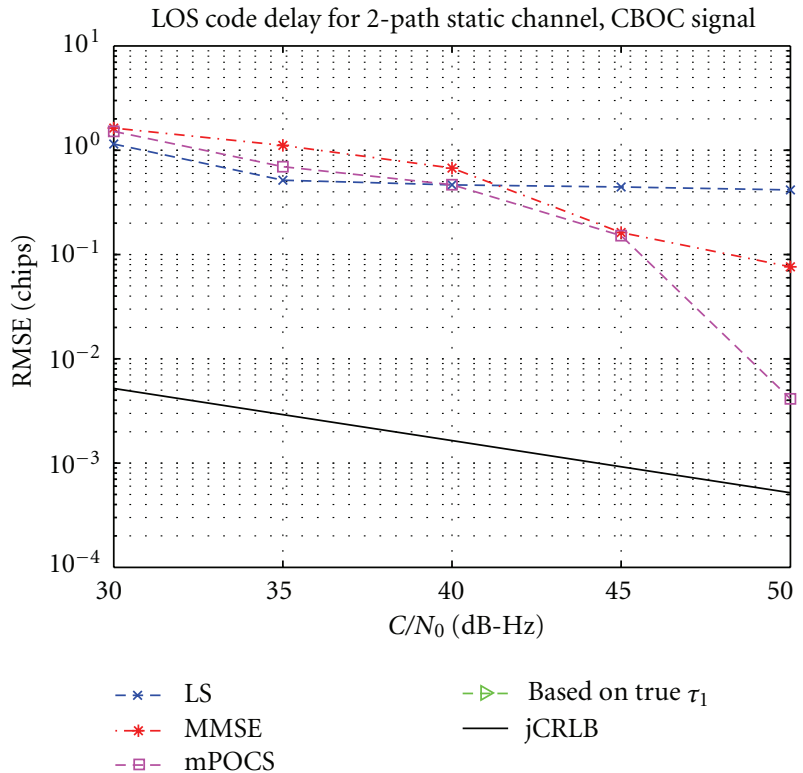

(b)

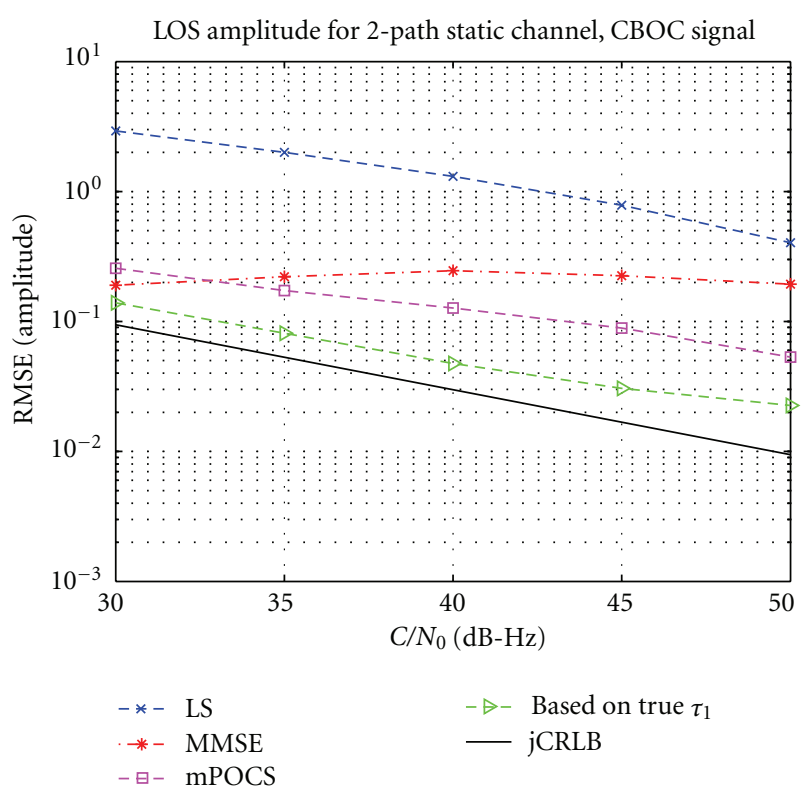

(c)

FIgURE 5: LOS carrier phase (a), code delay (b), and amplitude (c) versus $C / N_{0}$ for 2-path static channel and CBOC signal.

In the second part of our simulation results, we compare the theoretical limits with the performance of a set of deconvolution algorithms: the least square (LS), the minimum mean square error (MMSE), and our proposed modified POCS algorithm (here, for simplicity, we refer to it as "mPOCS," while in [24], it is denoted as "POCS2"). To briefly introduce it, mPOCS is an iterative deconvolution algorithm, which estimates jointly the LOS carrier phase and code delay and has been optimized for both Sine-BOC $(1,1)$ and BPSK modulated signals (the first modulation was the one initially proposed for the new Galileo OS signals, and the second modulation type is the one employed by the GPS coarse/acquisition (C/A) signals). Because both BOC and MBOC modulation types have been discussed in the context of GNSS specifications and since MBOC signals are also supposed to work with Sine-BOC receivers, the performance of mPOCS in the case of MBOC modulation will also show how flexible it is. Our POCS-based proposed algorithm is different from the previously proposed deconvolution approaches in two main ways: first, it incorporates some knowledge about the static multipath channel via estimated level crossing rates of receiver correlation function; second, it uses an adaptive threshold to reduce the various sources of interference (noise, multipath, and sidelobes in the autocorrelation 


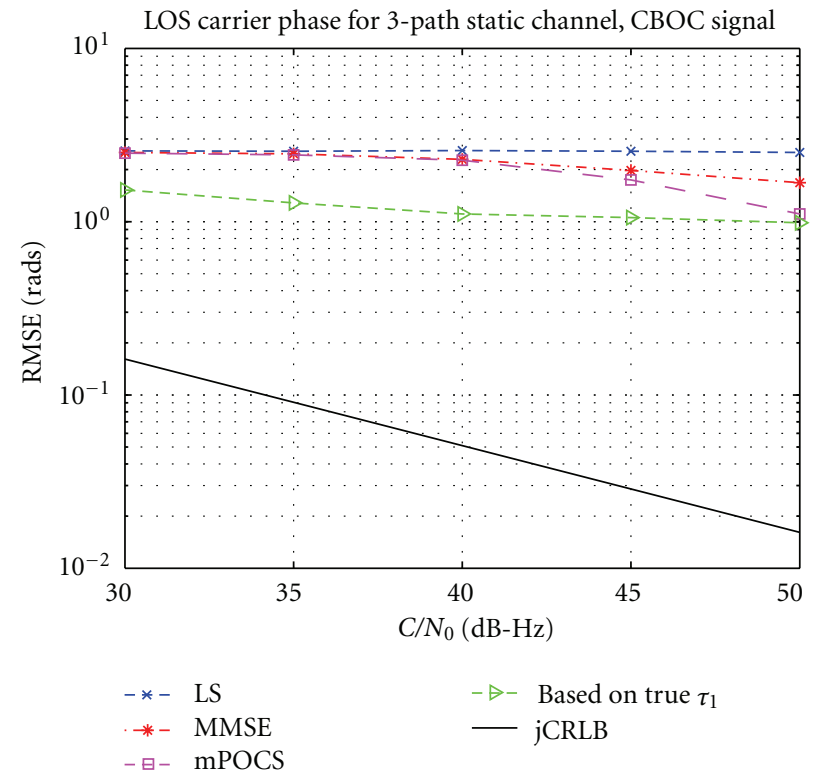

(a)

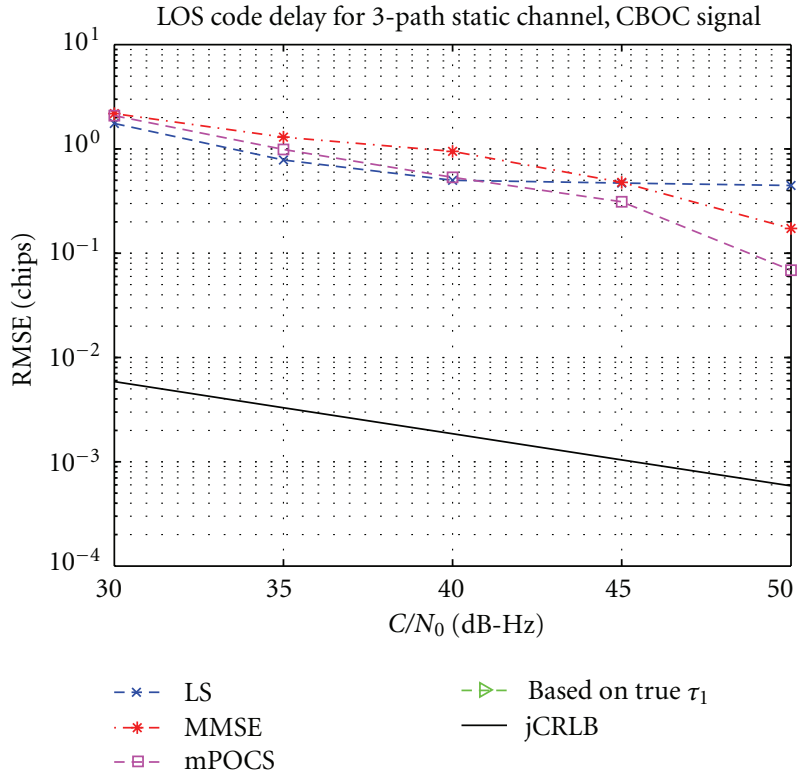

(b)

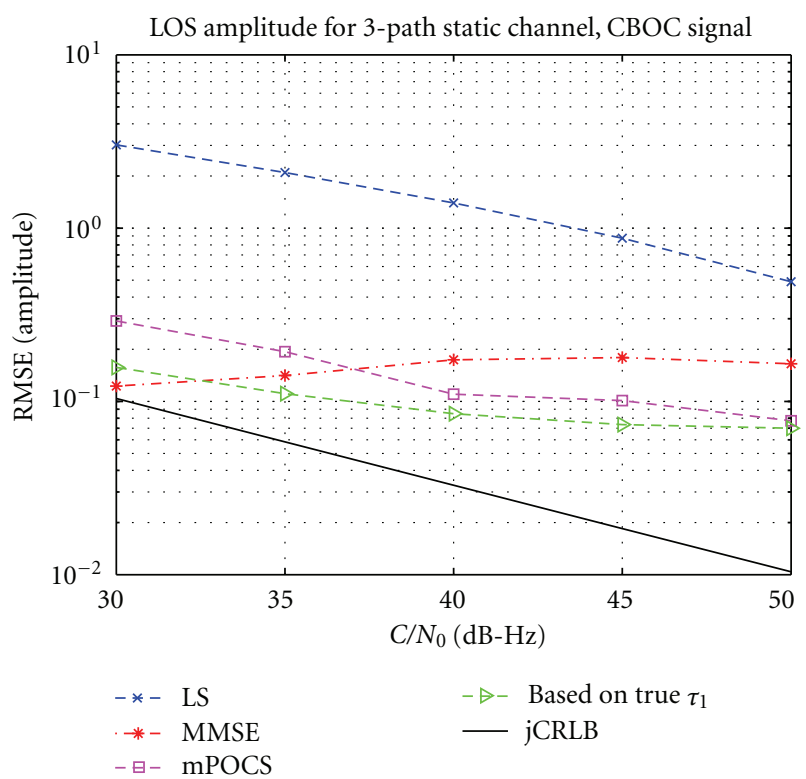

(c)

FIgURE 6: LOS carrier phase (a), code delay (b), and amplitude (c) versus $C / N_{0}$ for 3-path static channel and CBOC signal.

function of BOC-modulated signals). For brevity, we do not include a description of mPOCS algorithm. Instead, the interested reader is advised to refer to [24] for further details.

Because in this paper the estimation of all three synchronization parameters is considered, we incorporated into mPOCS the function of amplitude estimation as well (for the sake of completeness). More precisely, the amplitude of the LOS path is computed as $\alpha_{1}=\sqrt{\left|\hat{\mathbf{w}}\left(\hat{\tau}_{1}\right)\right|}$, where $\hat{\mathbf{w}}$ is the estimate of $\mathbf{w}$ and $\left(\hat{\tau}_{1}\right)$ is the estimate of code delay of the LOS path, both of which are outputs of the mPOCS algorithm. Except for the incorporation of the amplitude estimation in mPOCS, we have also made the following modification in our model compared to the one presented in [24]: each row of the pulse shape deconvolution matrix, $\mathbf{H}$, has been normalized as $\mathbf{H}(k,:)=\mathbf{H}(k,:) . / \max (\mathbf{H}(k,:))$, that is, normalized to one. We did this normalization because we found out that it improves the performance of the deconvolution algorithms. We also emphasize that the normalization of $\mathbf{H}$ takes place only when it is used by the deconvolution algorithms and not when the CRLB is computed.

Regarding the channel setup, we used similar decaying PDP model with the previously described unless otherwise stated. The oversampling factor $\left(N_{s}\right)$ was equal to 4 , and the processing of Acf is done in a window $\left(\tau_{\max }\right)$ of 4 chips length 


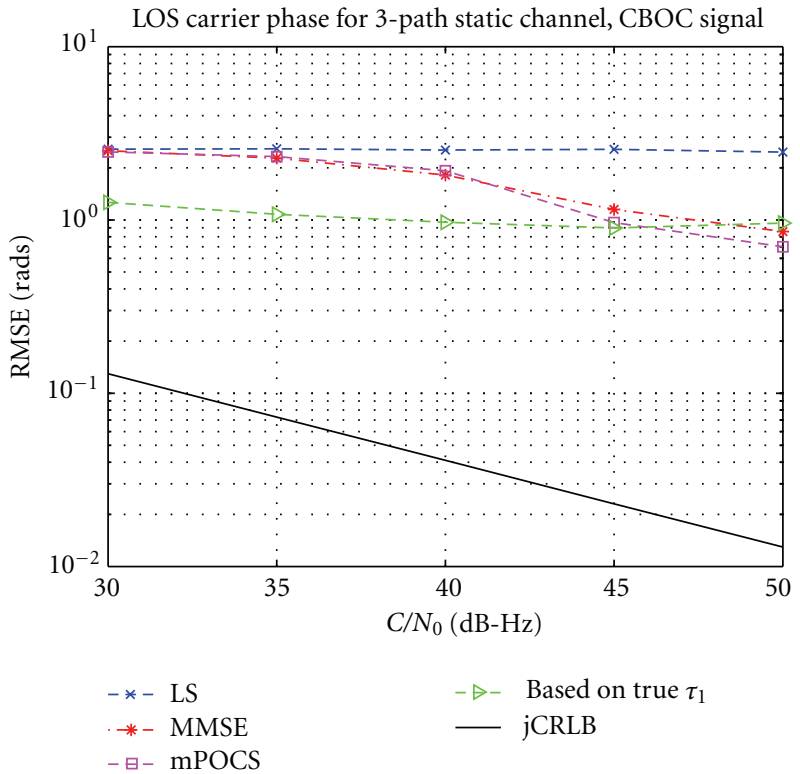

(a)

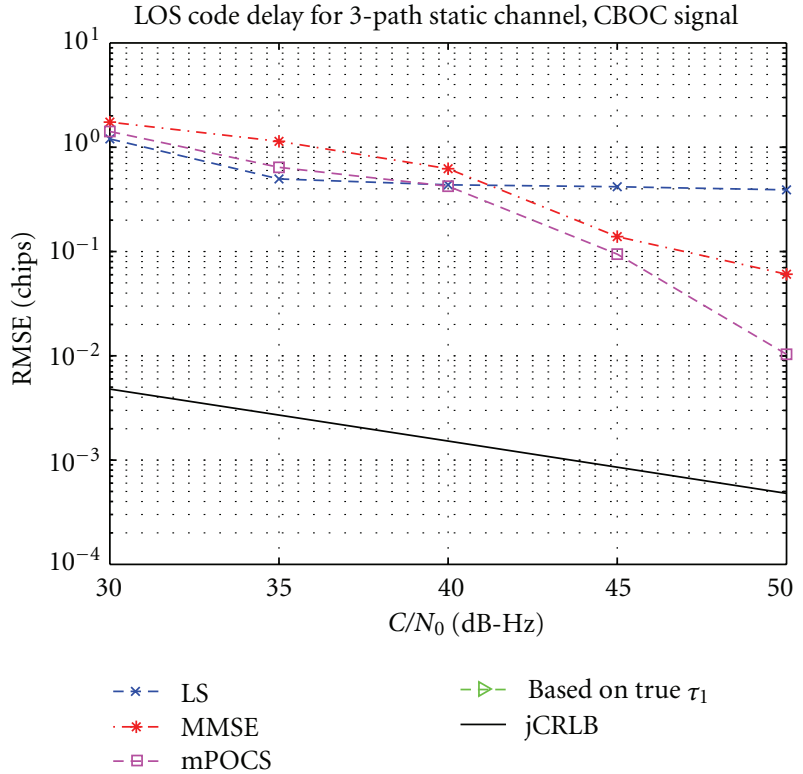

(b)

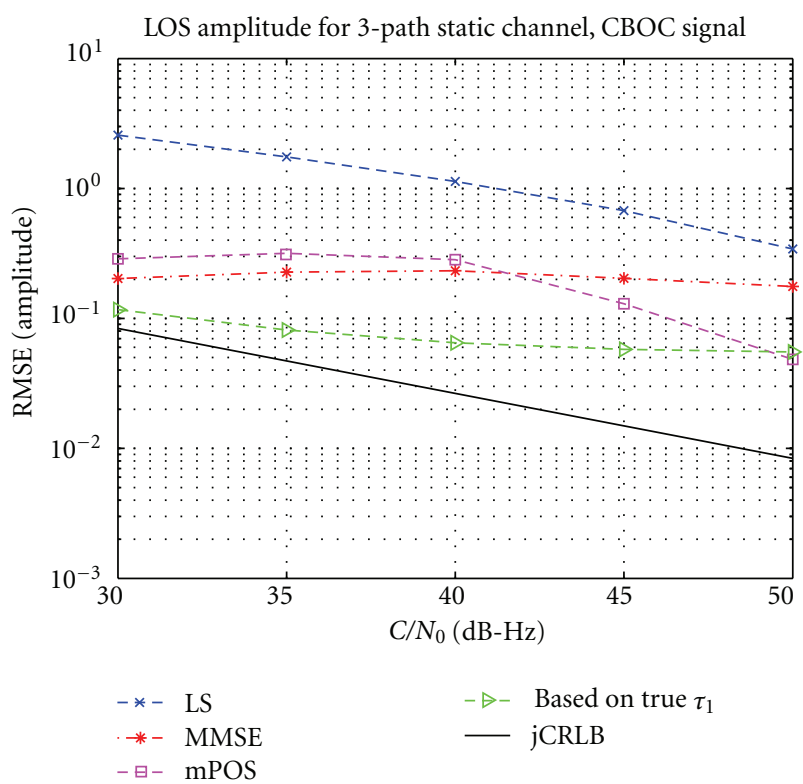

(c)

FIgURE 7: LOS carrier phase (a), code delay (b), and amplitude (c) versus $C / N_{0}$ for 3-path static channel and CBOC signal and $N_{c}=2$.

with a resolution of $1 /\left(N_{s} N B\right)$ chips (i.e., $\left.K=\tau_{\max } N_{s} N B\right)$. The coherent integration time $\left(N_{c}\right)$ was set to $10 \mathrm{~ms}$, while the noncoherent integration $\left(N_{\mathrm{nc}}\right)$ was performed in 1 blocks of $N_{c}$ length. We remark that because mPOCS estimates jointly the three synchronization parameters, we have used the corresponding joint CRLB (i.e., Case 4).

In Figure 5, we see the performance of the estimators versus $C / N_{0}$ in the case of two-path static channel. Among the deconvolution methods, mPOCS performs the best when $C / N_{0}$ is higher than $40 \mathrm{~dB}-\mathrm{Hz}$ for the phase and delay parameters, while for the amplitude parameter, mPOCs is better starting from $35 \mathrm{~dB}-\mathrm{Hz}$. We notice that in the case of delay parameter (top right plot), mPOCS is the one that converges faster than LS and MMSE towards CRLB. Figure 6 shows the results for the case of 3-path static channel. As in the case of 2-path scenario, LS has the worst average performance for all parameters, while mPOCS remains the best method for middle or higher $C / N_{0}$ values. When we increase the noncoherent integration from 1 to 2 , then the performance of the estimators is further improved (see Figure 7).

\section{Conclusions}

In this paper, we derived single and joint CRLBs for the unknown parameter vectors of carrier phase, code delay, and amplitude in multipath channel. Furthermore, we provided 
a theoretical analysis of the impact of different channel parameters such as $C / N_{0}$, path separation, and number of channel paths on the estimator accuracy bounds. Finally, we compared the performance between the derived theoretical limits and a set of deconvolution estimators, among which a modified projection onto convex set (mPOCS) algorithm [24]. All our experiments assumed CBOC modulation, which is the one selected for future Galileo OS signals. The simulations results show that mPOCS has the best performance among the other deconvolution methods for $\mathrm{C} / \mathrm{N}_{0}$ higher than 35 or $40 \mathrm{~dB}-\mathrm{Hz}$, depending on the signal parameter and the channel profile.

\section{Appendix}

For the computation of CRLB, we assume that the pdf satisfies the regularity conditions; that is,

$$
E\left[\frac{\partial \ln p(\tilde{\mathbf{y}} ; \boldsymbol{\theta})}{\partial \boldsymbol{\theta}}\right]=0 .
$$

In what follows, we use the logarithm of the likelihood function for calculating the Fischer information matrix (FIM). If we assume that the estimator $\hat{\boldsymbol{\theta}}$ is unbiased and that the pdf satisfies the regularity conditions, the CRLB for each of the four cases is found by inverting the corresponding FIM.

.1. CRLB for Single Vector Parameter. When computing the single CRLB, we assume that the other signal parameters are known or equivalently that they have been perfectly estimated. This assumption is made in order to eliminate the impact of the other signal parameters on the estimation bound of the parameter at hand, thus, leading to a "stricter" bound.

(a) Carrier Phase. Starting with the case in which the unknowns to be estimated are the carrier phases of the channel paths, we set $\boldsymbol{\theta}=\phi$. Differentiating once the loglikelihood function with respect to the unknown parameter vector $\phi$ gives

$$
\begin{aligned}
\frac{\partial \ln p(\tilde{\mathbf{y}} ; \phi)}{\partial \phi_{i}} & =-\frac{1}{2 \sigma^{2}} \sum_{k=1}^{K} \frac{\partial|\tilde{y}(k)-s(\phi ; k)|^{2}}{\partial \phi_{i}} \\
& =\frac{1}{\sigma^{2}} \sum_{k=1}^{K} \mathfrak{R}\left\{\frac{\partial s(\phi ; k)}{\partial \phi_{i}}\left[\tilde{y}^{*}(k)-s^{*}(\phi ; k)\right]\right\},
\end{aligned}
$$

where

$$
\begin{gathered}
\frac{\partial s(\phi ; k)}{\partial \phi_{i}}=j \alpha_{i} \tilde{h}\left(\tau_{i}, \tau_{k}\right) e^{j \phi_{i}}, \\
s^{*}(\phi ; k)=\sum_{l=1}^{L} \alpha_{l} \tilde{h}\left(\tau_{l}, \tau_{k}\right) e^{-j \phi_{l}}, \\
\tilde{y}^{*}(k)=\mathfrak{R}\{\tilde{y}(k)\}-j \mathfrak{I}\{\tilde{y}(k)\},
\end{gathered}
$$

and $\mathfrak{R}\{\cdot\}, \mathfrak{I}\{\cdot\}$ are used for denoting the real and the imaginary part, respectively. Substituting (A.3), (A.4), and (A.5) into (A.2) results in

$$
\begin{aligned}
\frac{\partial \ln p(\tilde{\mathbf{y}} ; \phi)}{\partial \phi_{i}}= & \frac{\alpha_{i}}{\sigma^{2}} \sum_{k=1}^{K} \tilde{h}\left(\tau_{i}, \tau_{k}\right) \\
& \times\left[-\mathfrak{R}\{\tilde{y}(k)\} \sin \left(\phi_{i}\right)+\mathfrak{I}\{\tilde{y}(k)\} \cos \left(\phi_{i}\right)\right. \\
& \left.+\sum_{l=1}^{L} \alpha_{l} \tilde{h}\left(\tau_{l}, \tau_{k}\right) \sin \left(\phi_{i}-\phi_{l}\right)\right] .
\end{aligned}
$$

Then, we compute the second derivatives for $\phi_{i}=\phi_{q}$ and $\phi_{i} \neq \phi_{q}$, respectively, as

$$
\begin{aligned}
& \frac{\partial^{2} \ln p(\tilde{\mathbf{y}} ; \phi)}{\partial \phi_{i}^{2}}= \frac{\alpha_{i}}{\sigma^{2}} \sum_{k=1}^{K} \tilde{h}\left(\tau_{i}, \tau_{k}\right) \\
& \times\left[-\mathfrak{R}\{\tilde{y}(k)\} \cos \left(\phi_{i}\right)-\mathfrak{I}\{\tilde{y}(k)\} \sin \left(\phi_{i}\right)\right. \\
&\left.+\sum_{\substack{l=1 \\
i \neq l}}^{L} \alpha_{i} \tilde{h}\left(\tau_{i}, \tau_{k}\right) \cos \left(\phi_{i}-\phi_{l}\right)\right] \\
& \frac{\partial^{2} \ln p(\tilde{\mathbf{y}} ; \phi)}{\partial \phi_{i} \partial \phi_{q}}=-\frac{\alpha_{i} \alpha_{q}}{\sigma^{2}} \sum_{k=1}^{K} \tilde{h}\left(\tau_{i}, \tau_{k}\right) \tilde{h}\left(\tau_{q}, \tau_{k}\right) \cos \left(\phi_{i}-\phi_{q}\right) .
\end{aligned}
$$

Notice that because the first two terms in the square brackets of (A.6) do not depend on $\phi_{i}$ or $\phi_{q}$, they are set to zero. In order to populate the Fischer information matrix (FIM), we distinguish between the elements located in the main diagonal (denoted as $[I(\phi)]_{i i}$ ) and the elements located outside it (denoted as $[I(\phi)]_{i q}$, for $i \neq q$ )

$$
\begin{aligned}
{[I(\phi)]_{i i} } & =-E\left[\frac{\partial^{2} \ln p(\tilde{\mathbf{y}} ; \phi)}{\partial \phi_{i}^{2}}\right] \\
& =\frac{\alpha_{i}}{\sigma^{2}} \sum_{k=1}^{K} \tilde{h}\left(\tau_{i}, \tau_{k}\right)
\end{aligned}
$$




$$
\begin{aligned}
& \times\left[E[\Re\{\tilde{y}(k)\}] \cos \left(\phi_{i}\right)\right. \\
& +E[\Im\{\tilde{I}(k)\}] \sin \left(\phi_{i}\right) \\
& \left.+\sum_{\substack{l=1 \\
i \neq l}}^{L} \alpha_{i} \tilde{h}\left(\tau_{i}, \tau_{k}\right) \cos \left(\phi_{i}-\phi_{l}\right)\right] \\
& =\frac{\alpha_{i}}{\sigma^{2}} \sum_{k=1}^{K} \tilde{h}\left(\tau_{i}, \tau_{k}\right) \\
& \times\left[\cos \left(\phi_{i}\right) \sum_{k=1}^{K} \alpha_{l} \tilde{h}\left(\tau_{l}, \tau_{k}\right) \cos \left(\phi_{l}\right)\right. \\
& +\sin \left(\phi_{i}\right) \sum_{k=1}^{K} \alpha_{l} \tilde{h}\left(\tau_{l}, \tau_{k}\right) \sin \left(\phi_{l}\right) \ldots \\
& \left.+\sum_{\substack{l=1 \\
i \neq l}}^{L} \alpha_{i} \tilde{h}\left(\tau_{i}, \tau_{k}\right) \cos \left(\phi_{i}-\phi_{l}\right)\right] \\
& =\frac{\alpha_{i}}{\sigma^{2}} \sum_{k=1}^{K} \tilde{h}\left(\tau_{i}, \tau_{k}\right) \\
& \times\left[\sum_{k=1}^{K} \alpha_{l} \tilde{h}\left(\tau_{l}, \tau_{k}\right) \cos \left(\phi_{i}-\phi_{l}\right)\right. \\
& \left.-\sum_{\substack{l=1 \\
i \neq l}}^{L} \alpha_{i} \tilde{h}\left(\tau_{i}, \tau_{k}\right) \cos \left(\phi_{i}-\phi_{l}\right)\right] \\
& =\frac{\alpha_{i}^{2}}{\sigma^{2}} \sum_{k=1}^{K} \tilde{h}^{2}\left(\tau_{i}, \tau_{k}\right) .
\end{aligned}
$$

Notice that due to the assumption of static channel, we have

$$
\begin{aligned}
E[\mathfrak{R}\{\tilde{y}(k)\}] & =E[\mathfrak{R}\{s(\phi ; k)\}]+E[\mathfrak{R}\{\tilde{v}(k)\}] \\
& =E\left[\mathfrak{R}\left\{\sum_{l=1}^{L} \alpha_{l} \tilde{h}\left(\tau_{l}, \tau_{k}\right) e^{j \phi_{l}}\right\}\right]+0 \\
& =E\left[\sum_{l=1}^{L} \alpha_{l} \tilde{h}\left(\tau_{l}, \tau_{k}\right) \cos \left(\phi_{l}\right)\right] \\
& =\sum_{l=1}^{L} \alpha_{l} \tilde{h}\left(\tau_{l}, \tau_{k}\right) \cos \left(\phi_{l}\right) .
\end{aligned}
$$

We remark that the model up to here is valid for both fading and static channels. For subsequent derivations, in case of a fading channel, we would need to compute $E\left[\alpha_{l} \cos \left(\phi_{l}\right)\right]$, where $\alpha_{l}$ would be distributed according to the fading channel profile (e.g., Rayleigh or Nakagami distributed) and $\phi_{l}$ is uniformly distributed over $[-\pi, \pi]$.Similarly, for the elements outside the main diagonal, we have

$$
\begin{aligned}
{[I(\phi)]_{i q} } & =-E\left[\frac{\partial^{2} p(\tilde{y} ; \phi)}{\partial \phi_{i} \phi_{q}}\right] \\
& =\frac{\alpha_{i} \alpha_{q}}{\sigma^{2}} \sum_{k=1}^{K} \tilde{h}\left(\tau_{i}, \tau_{k}\right) \tilde{h}\left(\tau_{q}, \tau_{k}\right) \cos \left(\phi_{i}-\phi_{q}\right) .
\end{aligned}
$$

Finally, the CRLB for the carrier phase of the $l$ th path is given by the element located in the $l$ th row and $l$ th column of the inverse Fischer information matrix

$$
\operatorname{var}\left(\hat{\phi}_{l}\right)=\left[I^{-1}(\phi)\right]_{l, l} \text {. }
$$

(b) Code Delay. Here, we have $\boldsymbol{\theta}=\boldsymbol{\tau}$. Differentiating once the log-likelihood function with respect to the unknown code delay gives

$$
\frac{\partial \ln p(\tilde{\mathbf{y}} ; \boldsymbol{\tau})}{\partial \tau_{i}}=\frac{1}{\sigma^{2}} \sum_{k=1}^{K} \Re\left\{\frac{\partial s(\boldsymbol{\tau} ; k)}{\partial \tau_{i}}\left[\tilde{y}^{*}(k)-s^{*}(\boldsymbol{\tau} ; k)\right]\right\},
$$

where

$$
\frac{\partial s(\boldsymbol{\tau} ; k)}{\partial \tau_{i}}=\alpha_{i} \tilde{h}^{\prime}\left(\tau_{i}, \tau_{k}\right) e^{j \phi_{i}}
$$

Now, we substitute (A.4), (A.5), and (A.13) into (A.12), and we get

$$
\begin{aligned}
& \frac{\partial \ln p(\tilde{\mathbf{y}} ; \boldsymbol{\tau})}{\partial \tau_{i}} \\
&=\frac{\alpha_{i}}{\sigma^{2}} \sum_{k=1}^{K} \tilde{h}^{\prime}\left(\tau_{i}, \tau_{k}\right)\left[\Re\{\tilde{y}(k)\} \cos \left(\phi_{i}\right)+\Im\{\tilde{y}(k)\} \sin \left(\phi_{i}\right)\right. \\
& \\
&\left.\quad-\sum_{l=1}^{L} \alpha_{l} \tilde{h}\left(\tau_{l}, \tau_{k}\right) \cos \left(\phi_{i}-\phi_{l}\right)\right]
\end{aligned}
$$

Then, we calculate the second derivatives by distinguishing between differentiation with the same path or not. After some mathematical manipulation, we get

$$
\begin{aligned}
& \frac{\partial^{2} \ln p(\tilde{\mathbf{y}} ; \boldsymbol{\tau})}{\partial \tau_{i}^{2}} \\
& =\frac{\alpha_{i}}{\sigma^{2}} \sum_{k=1}^{K} \tilde{h}^{\prime \prime}\left(\tau_{i}, \tau_{k}\right)\left[\Re\{\tilde{y}(k)\} \cos \left(\phi_{i}\right)+\Im\{\tilde{y}(k)\} \sin \left(\phi_{i}\right)\right. \\
& \left.\quad-\sum_{l=1}^{L} \alpha_{l} \tilde{h}\left(\tau_{l}, \tau_{k}\right) \cos \left(\phi_{i}-\phi_{l}\right)\right] \\
& -\frac{a_{i}^{2} \sum^{2}}{\sigma_{k=1}^{K}}\left[\tilde{h}^{\prime}\left(\tau_{i}, \tau_{k}\right)\right]^{2}
\end{aligned}
$$


Because the first two terms in the square brackets of (A.14) do not depend on the differentiating parameter, they are ignored, and we get

$$
\frac{\partial^{2} \ln p(\tilde{\mathbf{y}} ; \boldsymbol{\tau})}{\partial \tau_{i} \partial \tau_{q}}=-\frac{\alpha_{i} \alpha_{q}}{\sigma^{2}} \sum_{k=1}^{K} \tilde{h}^{\prime}\left(\tau_{i}, \tau_{k}\right) \tilde{h}\left(\tau_{q}, \tau_{k}\right) \cos \left(\phi_{i}-\phi_{q}\right) .
$$

Using (A.15) and (A.16), we find that the FIM elements are

$$
\begin{aligned}
& {[I(\boldsymbol{\tau})]_{i i}=-E\left[\frac{\partial^{2} \ln p(\tilde{\mathbf{y}} ; \tau)}{\partial \tau_{i}^{2}}\right]} \\
& =\frac{a_{i}}{\sigma^{2}} \sum_{k=1}^{K} \tilde{h}^{\prime \prime}\left(\tau_{i}, \tau_{k}\right) \\
& \times\left[-\cos \left(\phi_{i}\right) \sum_{k=1}^{K} \alpha_{l} \tilde{h}\left(\tau_{l}, \tau_{k}\right) \cos \left(\phi_{l}\right)\right. \\
& -\sin \left(\phi_{i}\right) \sum_{k=1}^{K} \alpha_{l} \tilde{h}\left(\tau_{l}, \tau_{k}\right) \sin \left(\phi_{l}\right) \cdots \\
& \left.+\sum_{k=1}^{K} \alpha_{l} \tilde{h}\left(\tau_{l}, \tau_{k}\right) \cos \left(\phi_{i}-\phi_{l}\right)\right] \\
& +\frac{a_{i}^{2}}{\sigma^{2}} \sum_{k=1}^{K}\left[\tilde{h}^{\prime}\left(\tau_{i}, \tau_{k}\right)\right]^{2} \\
& =-\frac{a_{i}}{\sigma^{2}} \sum_{k=1}^{K} \widetilde{h}^{\prime \prime}\left(\tau_{i}, \tau_{k}\right) \sum_{k=1}^{K} \alpha_{l} \widetilde{h}\left(\tau_{l}, \tau_{k}\right) \\
& \times\left[\cos \left(\phi_{i}-\phi_{l}\right)-\cos \left(\phi_{i}-\phi_{l}\right)\right] \\
& +\frac{a_{i}^{2}}{\sigma^{2}} \sum_{k=1}^{K}\left[\tilde{h}^{\prime}\left(\tau_{i}, \tau_{k}\right)\right]^{2} \\
& =\frac{a_{i}^{2}}{\sigma^{2}} \sum_{k=1}^{K}\left[\tilde{h}^{\prime}\left(\tau_{i}, \tau_{k}\right)\right]^{2}, \\
& {[I(\boldsymbol{\tau})]_{i q}=-E\left[\frac{\partial^{2} \ln p(\tilde{\mathbf{y}} ; \tau)}{\partial \tau_{i} \partial \tau_{q}}\right]} \\
& =\frac{\alpha_{i} \alpha_{q}}{\sigma^{2}} \sum_{k=1}^{K} \tilde{h}^{\prime}\left(\tau_{i}, \tau_{k}\right) \tilde{h}\left(\tau_{q}, \tau_{k}\right) \cos \left(\phi_{i}-\phi_{q}\right) \text {. }
\end{aligned}
$$

Finally, the CRLB for the code delay of each path is taken from the diagonal of the inverse FIM as

$$
\operatorname{var}\left(\hat{\tau}_{l}\right)=\left[I^{-1}(\boldsymbol{\tau})\right]_{l, l} .
$$

(c) Amplitude. After we differentiate the log-likelihood function with respect to the amplitude of the $i$ th path, we get

$$
\frac{\partial \ln p(\tilde{\mathbf{y}} ; \boldsymbol{\alpha})}{\partial \alpha_{i}}=\frac{1}{\sigma^{2}} \sum_{k=1}^{K} \mathfrak{R}\left\{\frac{\partial s(\boldsymbol{\alpha} ; k)}{\partial \alpha_{i}}\left[\tilde{y}^{*}(k)-s^{*}(\boldsymbol{\alpha} ; k)\right]\right\},
$$

where

$$
\frac{\partial s(\boldsymbol{\alpha} ; k)}{\partial \alpha_{i}}=\tilde{h}\left(\tau_{i}, \tau_{k}\right) e^{j \phi_{i}} .
$$

Substituting (A.4), (A.5), and (A.20) into (A.19), and after some mathematical manipulations, we get

$$
\begin{aligned}
\frac{\partial \ln p(\tilde{\mathbf{y}} ; \boldsymbol{\alpha})}{\partial \alpha_{i}}= & \frac{1}{\sigma^{2}} \sum_{k=1}^{K} \tilde{h}\left(\tau_{i}, \tau_{k}\right) \\
\times & {\left[\cos \phi_{i} \Re\{\tilde{y}(k)\}+\sin \phi_{i} \Im\{\tilde{y}(k)\}\right.} \\
& \left.\quad-\sum_{l=1}^{L} \alpha_{l} \tilde{h}\left(\tau_{l}, \tau_{k}\right) \cos \left(\phi_{i}-\phi_{l}\right)\right] .
\end{aligned}
$$

Because the first two terms inside the square brackets of (A.21) do not depend on the amplitude parameter, the differentiation of them leads to zero, and the final results for the second derivatives are

$$
\begin{gathered}
\frac{\partial^{2} \ln p(\tilde{\mathbf{y}} ; \boldsymbol{\alpha})}{\partial \alpha_{i}^{2}}=-\frac{1}{\sigma^{2}} \sum_{k=1}^{K} \tilde{h}^{2}\left(\tau_{i}, \tau_{k}\right), \\
\frac{\partial^{2} \ln p(\tilde{\mathbf{y}} ; \boldsymbol{\alpha})}{\partial \alpha_{i} \partial \alpha_{q}}=-\frac{1}{\sigma^{2}} \sum_{k=1}^{K} \tilde{h}\left(\tau_{i}, \tau_{k}\right) \tilde{h}\left(\tau_{q}, \tau_{k}\right) \cos \left(\phi_{i}-\phi_{q}\right),
\end{gathered}
$$

and the FIM elements

$$
\begin{gathered}
{[I(\boldsymbol{\alpha})]_{i i}=\frac{1}{\sigma^{2}} \sum_{k=1}^{K} \tilde{h}^{2}\left(\tau_{i}, \tau_{k}\right),} \\
{[I(\boldsymbol{\alpha})]_{i q}=\frac{1}{\sigma^{2}} \sum_{k=1}^{K} \widetilde{h}\left(\tau_{i}, \tau_{k}\right) \widetilde{h}\left(\tau_{q}, \tau_{k}\right) \cos \left(\phi_{i}-\phi_{q}\right) .}
\end{gathered}
$$

Finally, the CRLB for the amplitude of the $l$ th path is

$$
\operatorname{var}\left(\hat{\alpha}_{l}\right)=\left[I^{-1}(\boldsymbol{\alpha})\right]_{l, l} .
$$

A.2. CRLB for Joint Vector Parameters. Here, we derive the joint CRLB for all possible combinations of the unknown parameter vectors. First, we consider the case of joint carrier phase and code delay estimation (from now on, this case will be referred to as Case 1). Second, we have the case of joint carrier phase and amplitude estimation (Case 2). Third, the case of joint code delay and amplitude (Case 3 ) and last the case of jointly estimating all three parameter vectors (Case 4). We also remind the reader that in all cases where two parameter vectors are jointly estimated, we assume that the third parameter vector is known or perfectly estimated.

Case 1 ((C1)-Carrier Phase and Code Delay). The $2 \times 2$ joint FIM is given by

$$
I_{1, \text { joint }}(\phi, \boldsymbol{\tau})=\left[\begin{array}{cc}
I(\phi) & I(\phi, \boldsymbol{\tau}) \\
I(\boldsymbol{\tau}, \phi) & I(\boldsymbol{\tau})
\end{array}\right],
$$


where $I(\phi)$ and $I(\boldsymbol{\tau})$ can be constructed using (A.8), (A.10), and (A.17). Moreover, after some mathematical manipulations, we have

$$
\begin{gathered}
{[I(\phi, \tau)]_{i q}=-\frac{\alpha_{i} \alpha_{q}}{\sigma^{2}} \sum_{k=1}^{K} \tilde{h}\left(\tau_{i}, \tau_{k}\right) \tilde{h}^{\prime}\left(\tau_{q}, \tau_{k}\right) \sin \left(\phi_{i}-\phi_{q}\right),} \\
{[I(\tau, \phi)]_{i q}=\frac{\alpha_{i} \alpha_{j}}{\sigma^{2}} \sum_{k=1}^{K} \tilde{h}^{\prime}\left(\tau_{i}, \tau_{k}\right) \tilde{h}\left(\tau_{q}, \tau_{k}\right) \sin \left(\phi_{i}-\phi_{q}\right),}
\end{gathered}
$$

where it can be proven that for $i=j$, we have $[I(\phi, \tau)]_{i i}=$ $[I(\tau, \phi)]_{i i}=0$. Now, we can obtain the CRLBs for each vector parameter from the diagonal elements of the inverse FIM as

$$
\begin{gathered}
\operatorname{var}_{1, \text { joint }}(\hat{\phi})=\left[\operatorname{diag}\left(I_{1, \text { joint }}^{-1}(\phi, \boldsymbol{\tau})\right)\right]_{1: L}, \\
\operatorname{var}_{1, \text { joint }}(\hat{\boldsymbol{\tau}})=\left[\operatorname{diag}\left(I_{1, \text { joint }}^{-1}(\phi, \boldsymbol{\tau})\right)\right]_{L+1: 2 L} .
\end{gathered}
$$

Case 2 ((C2) - Carrier Phase and Amplitude). Similarly with Case 1, the joint FIM is given by

$$
I_{2, \text { joint }}(\phi, \boldsymbol{\alpha})=\left[\begin{array}{cc}
I(\phi) & I(\phi, \boldsymbol{\alpha}) \\
I(\boldsymbol{\alpha}, \phi) & I(\boldsymbol{\alpha})
\end{array}\right],
$$

where $I(\phi)$ and $I(\boldsymbol{\alpha})$ can be formed using (A.8), (A.10), and (A.23). Also, we have

$$
\begin{gathered}
{[I(\phi, \boldsymbol{\alpha})]_{i q}=-\frac{\alpha_{i}}{\sigma^{2}} \sum_{k=1}^{K} \tilde{h}\left(\tau_{i}, \tau_{k}\right) \tilde{h}\left(\tau_{q}, \tau_{k}\right) \sin \left(\phi_{i}-\phi_{q}\right),} \\
{[I(\boldsymbol{\alpha}, \phi)]_{i q}=\frac{\alpha_{j}}{\sigma^{2}} \sum_{k=1}^{K} \tilde{h}\left(\tau_{i}, \tau_{k}\right) \tilde{h}\left(\tau_{q}, \tau_{k}\right) \sin \left(\phi_{i}-\phi_{q}\right),}
\end{gathered}
$$

where for $i=q$, we get $[I(\phi, \boldsymbol{\alpha})]_{i i}=[I(\boldsymbol{\alpha}, \phi)]_{i i}=0$. Similarly, the CRLBs can be found as

$$
\begin{aligned}
\operatorname{var}_{2, \text { joint }}(\hat{\phi}) & =\left[\operatorname{diag}\left(I_{2, \text { joint }}^{-1}(\phi, \boldsymbol{\alpha})\right)\right]_{1: L^{\prime}} \\
\operatorname{var}_{2, \text { joint }}(\hat{\boldsymbol{\alpha}}) & =\left[\operatorname{diag}\left(I_{2, \text { joint }}^{-1}(\phi, \boldsymbol{\alpha})\right)\right]_{L+1: 2 L}
\end{aligned}
$$

Case 3 ((C3)_Code Delay and Amplitude). For this case, the FIM is formed as

$$
I_{3, \text { joint }}(\boldsymbol{\tau}, \boldsymbol{\alpha})=\left[\begin{array}{cc}
I(\boldsymbol{\tau}) & I(\boldsymbol{\tau}, \boldsymbol{\alpha}) \\
I(\boldsymbol{\alpha}, \boldsymbol{\tau}) & I(\boldsymbol{\alpha})
\end{array}\right],
$$

where $I(\boldsymbol{\tau})$ and $I(\boldsymbol{\alpha})$ can be found using (A.8), (A.10), and (A.23). Furthermore, we have

$$
\begin{gathered}
{[I(\boldsymbol{\tau}, \boldsymbol{\alpha})]_{i i}=\frac{\alpha_{i}}{\sigma^{2}} \sum_{k=1}^{K} \tilde{h}^{\prime}\left(\tau_{i}, \tau_{k}\right) \tilde{h}\left(\tau_{q}, \tau_{k}\right),} \\
{[I(\boldsymbol{\tau}, \boldsymbol{\alpha})]_{i q}=\frac{\alpha_{i}}{\sigma^{2}} \sum_{k=1}^{K} \tilde{h}^{\prime}\left(\tau_{i}, \tau_{k}\right) \tilde{h}\left(\tau_{q}, \tau_{k}\right) \cos \left(\phi_{i}-\phi_{q}\right),} \\
{[I(\boldsymbol{\alpha}, \boldsymbol{\tau})]_{i i}=\frac{\alpha_{q}}{\sigma^{2}} \sum_{k=1}^{K} \tilde{h}\left(\tau_{i}, \tau_{k}\right) \tilde{h}^{\prime}\left(\tau_{q}, \tau_{k}\right),} \\
{[I(\boldsymbol{\alpha}, \boldsymbol{\tau})]_{i q}=\frac{\alpha_{q}}{\sigma^{2}} \sum_{k=1}^{K} \tilde{h}\left(\tau_{i}, \tau_{k}\right) \tilde{h}^{\prime}\left(\tau_{q}, \tau_{k}\right) \cos \left(\phi_{i}-\phi_{q}\right) .}
\end{gathered}
$$

The CRLBs for each vector parameter are

$$
\begin{aligned}
& \operatorname{var}_{3, \text { joint }}(\hat{\boldsymbol{\alpha}})=\left[\operatorname{diag}\left(I_{3, \text { joint }}^{-1}(\boldsymbol{\alpha}, \boldsymbol{\tau})\right)\right]_{1: L}, \\
& \operatorname{var}_{3, \text { joint }}(\hat{\boldsymbol{\tau}})=\left[\operatorname{diag}\left(I_{3, \text { joint }}^{-1}(\boldsymbol{\alpha}, \boldsymbol{\tau})\right)\right]_{L+1: 2 L}
\end{aligned}
$$

Case 4 ((C4) - Carrier Phase, Code Delay and Amplitude). The joint FIM for the case where all three vector parameters are to be estimated as

$$
I_{4, \text { joint }}(\phi, \boldsymbol{\tau}, \boldsymbol{\alpha})=\left[\begin{array}{ccc}
I(\phi) & I(\phi, \boldsymbol{\tau}) & I(\phi, \boldsymbol{\alpha}) \\
I(\boldsymbol{\tau}, \phi) & I(\boldsymbol{\tau}) & I(\boldsymbol{\tau}, \boldsymbol{\alpha}) \\
I(\boldsymbol{\alpha}, \phi) & I(\boldsymbol{\alpha}, \tau) & I(\boldsymbol{\alpha})
\end{array}\right],
$$

where its diagonal elements can be found from Part A and the rest of the elements have been already derived in Cases 1, 2, and 3. Finally, the CRLBs for each of the unknown parameter vectors can be found according to

$$
\begin{aligned}
\operatorname{var}_{4, \text { joint }}(\hat{\phi}) & =\left[\operatorname{diag}\left(I_{\text {joint }}^{-1}(\phi, \boldsymbol{\tau}, \boldsymbol{\alpha})\right)\right]_{1: L^{\prime}}, \\
\operatorname{var}_{4, \text { joint }}(\hat{\boldsymbol{\tau}}) & =\left[\operatorname{diag}\left(I_{\text {joint }}^{-1}(\phi, \boldsymbol{\tau}, \boldsymbol{\alpha})\right)\right]_{L+1: 2 L}, \\
\operatorname{var}_{4, \text { joint }}(\hat{\boldsymbol{\alpha}}) & =\left[\operatorname{diag}\left(I_{\text {joint }}^{-1}(\phi, \boldsymbol{\tau}, \boldsymbol{\alpha})\right)\right]_{2 L+1: 3 L}
\end{aligned}
$$

\section{Acknowledgments}

This work has been supported by the Tampere Doctoral Program in Information Science and Engineering (TISE) and by the Academy of Finland. The work of A. H. Sayed was supported in part by NSF Grant no. ECS-0725441.

\section{References}

[1] E. Kaplan, Understanding GPS: Principles and Applications, Artech House, Norwood, Mass, USA, 1996.

[2] M. Braasch, Multipath Effects, Global Positioning Systems: Theory and Applications, vol. 1, chapter 14, American Institute of Aeronautics and Astronautics, 1996.

[3] B. Townsend and P. Fenton, "Practical approach to the reduction of pseudorange multipath errors in a L1 GPS receiver," in Proceedings of the 7th International Technical Meeting of the Satellite Division of the Institute of Navigation (ION-GPS '94), vol. 1, pp. 143-148, Salt Lake City, Utah, USA, September 1994.

[4] A. J. van Dierendonck, P. Fenton, and T. Ford, "Theory and performance of narrow correlator spacing in a GPS receiver," Navigation, Journal of the Institute of Navigation, vol. 39, no. 3, pp. 265-283, 1992.

[5] G. McGraw and M. Braasch, "GNSS multipath mitigation using high resolution correlator concepts," in Proceedings of the ION National Technical Meeting, pp. 333-342, San Diego, Calif, USA, January 1999.

[6] V. A. Veitsel, A. Zhdanov, and M. I. Zhodzishsky, "The mitigation of multipath errors by strobe correlators in GPS/GLONASS receivers," GPS Solutions, 1998.

[7] D. Skournetou and E. S. Lohan, "Non-coherent multiple correlator delay structures and their tracking performance for Galileo signals," in Proceedings of the European Navigation Conference in Global Navigation Satellite Systems (ENC-GNSS '07), pp. 247-258, Geneva, Switzerland, 2007. 
[8] E. S. Lohan, A. Lakhzouri, and M. Renfors, "Feedforward delay estimators in adverse multipath propagation for galileo and modernized GPS signals," EURASIP Journal on Applied Signal Processing, vol. 2006, Article ID 50971, 19 pages, 2006.

[9] M. Z. H. Bhuiyan, E. S. Lohan, and M. Renfors, "Peak tracking algorithm for Galileo-based positioning in multipath fading channels," in Proceedings of the IEEE International Conference on Communications (ICC '07), pp. 5927-5932, Glasgow, Scotland, UK, June 2007.

[10] D. Skournetou and E. S. Lohan, "Discontinuity-based code delay estimator for GNSS signals," in Proceedings of the 4th Advanced Satellite Mobile Systems (ASMS '08), pp. 213-222, Bologna, Italy, August 2008.

[11] M. S. Braasch and A. J. van Dierendonck, "GPS receiver architectures and measurements," Proceedings of the IEEE, vol. 87, no. 1, pp. 48-64, 1999.

[12] S. K. Kalyanaraman, J. M. Kelly, M. S. Braasch, and J. Kacirek, "Influence of GPS code tracking on carrier-phase multipath performance," in Proceeding of the IEEE Aerospace Conference Proceedings, pp. 1677-1686, March 2004.

[13] S. K. Kalyanaraman, M. S. Braasch, and J. M. Kelly, "Code tracking architecture influence on GPS carrier multipath," IEEE Transactions on Aerospace and Electronic Systems, vol. 42, no. 2, pp. 548-561, 2006.

[14] J. K. Ray and M. E. Cannon, "Characterization of GPS carrier phase multipath," in Proceedings of the Institute of Navigation National Technical Meeting (ION NTM '99), pp. 343-352, San Diego, Calif, USA, January 1999.

[15] L. Garin and J. Rousseau, "Enhanced strobe correlator multipath rejection for code and carrier," in Proceedings of the 10th International Technical Meeting of the Satellite Division of the Institute of Navigation (ION-GPS '97), pp. 559-568, Kansas City, Mo, USA, September 1997.

[16] B. R. Townsend, P. C. Fenton, K. J. V. Dierendonck, and D. J. R. van Nee, "Ll carrier phase multipath error reduction using medll technology," in Proceedings of the 8th International Technical Meeting of the Satellite Division of the Institute of Navigation (ION-GPS '95), Palm Springs, Calif, USA, September, 1995.

[17] J. W. Betz, "The offset carrier modulation for GPS modernization," in Proceedings of the Institute of Navigation (ION) Technical Meeting, pp. 639-648, 1999.

[18] "Galileo Open Service signal in space interface control document OS SIS ICS," September 2010, http://ec.europa.eu/ enterprise/policies/satnav/galileo/open-service/index_en.htm.

[19] A. Jovanovic, Y. Tawk, C. Botteron, and P. A. Farine, "Multipath mitigation techniques for CBOC, TMBOC and ALtBOC signals using advanced correlators architectures," in Proceedings of the IEEE/ION Position, Location And Navigation Symposium, pp. 1127-1136, Indian Wells/Palm Springs, Calif, USA, May 2010.

[20] A. Jovanovic, C. Botteron, and P. A. Farine, "Analysis and performance of tracking schemes for galileo mboc signal," in Proceedings of the European Navigation Conference on Global Navigation Satellite Systems (ENC-GNSS '09), Naples, Italy, May 2009.

[21] M. Fantino, G. Marucco, P. Mulassano, and M. Pini, "Performance analysis of MBOC, AltBOC and BOC modulations in terms of multipath effects on the carrier tracking loop within GNSS receivers," in Proceedings of the IEEE/ION Position, Location and Navigation Symposium, PLANS, pp. 369-376, Monterey, Calif, USA, May 2008.

[22] M. Fantino, P. Mulassano, F. Dovis, and L. Lo Presti, "Performance of the proposed galileo CBOC modulation in heavy multipath environment," Wireless Personal Communications, vol. 44, no. 3, pp. 323-339, 2008.
[23] N. R. Yousef, A. H. Sayed, and N. Khajehnouri, "Adaptive subchip multipath resolving for wireless location systems," EURASIP Journal on Applied Signal Processing, vol. 2006, Article ID 25431, 16 pages, 2006.

[24] D. Skournetou, A. H. Sayed, and E. S. Lohan, "A deconvolution algorithm for estimating jointly the line-of-sight code delay and carrier phase of GNSS signals," in Proceedings of the European Navigation Conference on Global Navigation Satellite Systems (ENC-GNSS '09), Naples, Italy, May 2009.

[25] S. M. Kay, Fundamentals of Statistical Signal Processing, vol. 2, Prentice Hall, New York, NY, USA, 1993.

[26] E. S. Lohan, A. Lakhzouri, and M. Renfors, "Binary-offsetcarrier modulation techniques with applications in satellite navigation systems," Wiley Journal of Wireless Communications and Mobile Computing, vol. 7, no. 6, pp. 767-779, 2007.

[27] E. A. Gadallah, Global Positioning System (GPS) Receiver Design For Multipaths Mitigation, Ph.D. thesis, Air Force Institute of Technology, Wright-Patterson American Foundation for the Blind (AFB), Ohio, USA, 1998.

[28] M. Irsigler, J. A. Avila-Rodriguez, and G. W. Hein, "Criteria for GNSS multipath performance assessment," in Proceedings of the International Technical Meeting of the Institute of Navigation (ION-GNSS '05), Long Beach, Calif, USA, September 2005. 

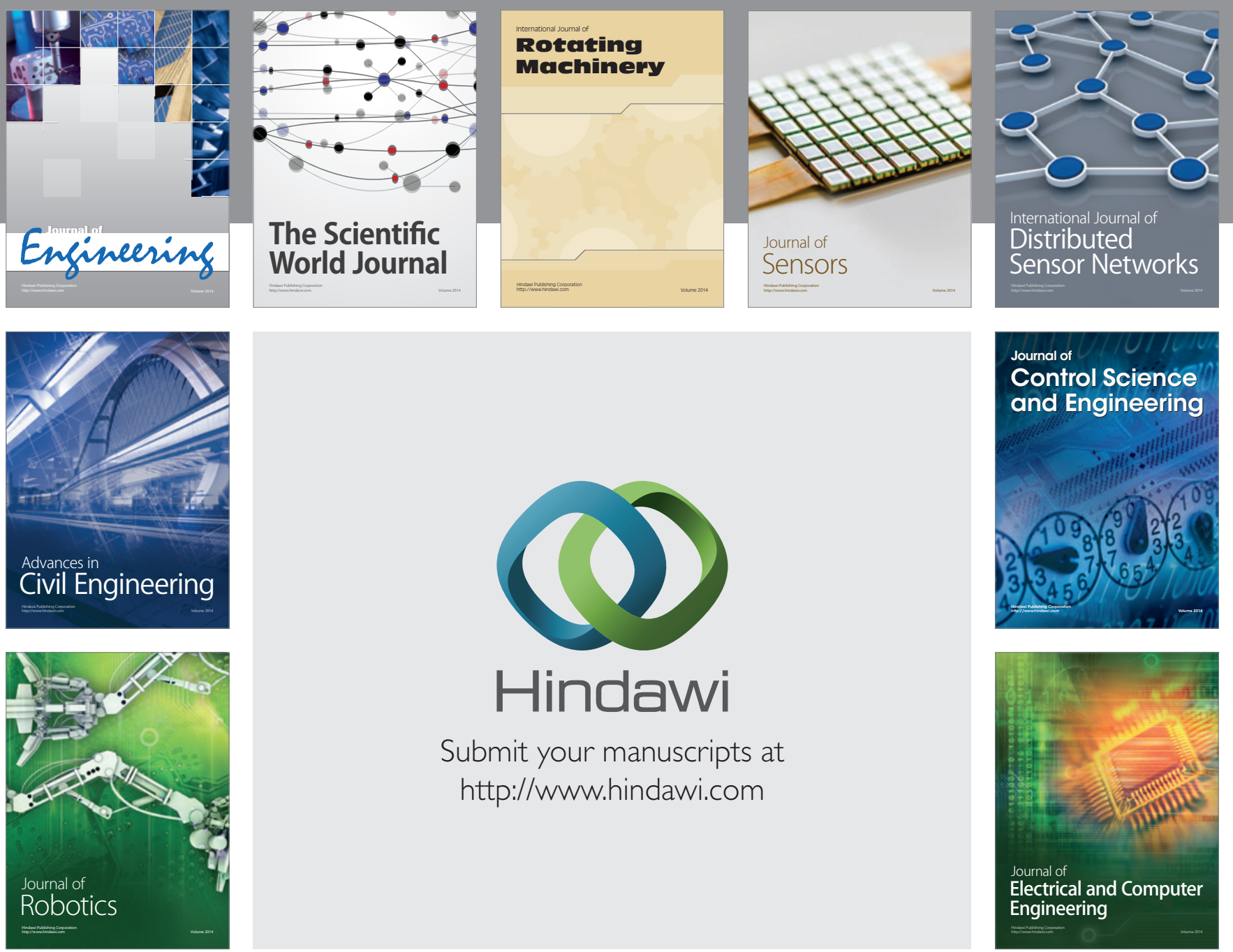

Submit your manuscripts at

http://www.hindawi.com
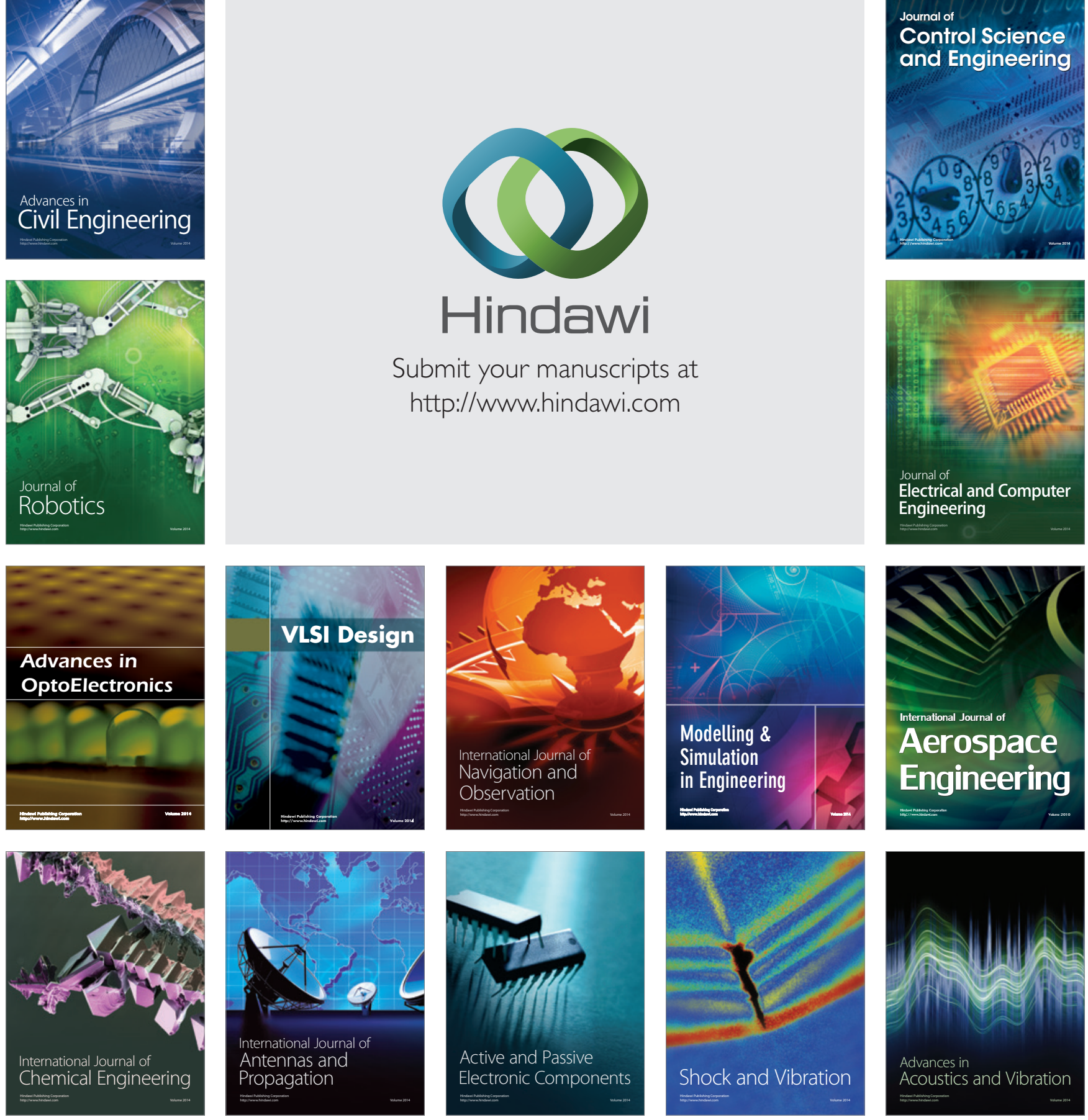